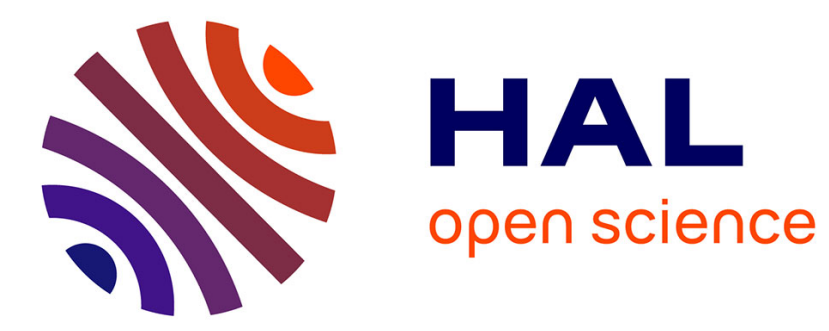

\title{
On some k-scoring rules for committee elections: agreement and Condorcet Principle
}

\author{
Mostapha Diss, Eric Kamwa, Abdelmonaim Tlidi
}

\section{To cite this version:}

Mostapha Diss, Eric Kamwa, Abdelmonaim Tlidi. On some k-scoring rules for committee elections: agreement and Condorcet Principle. Revue d'Economie Politique, 2020, 130 (5), pp.699-726. hal02147735

\section{HAL Id: hal-02147735 \\ https://hal.univ-antilles.fr/hal-02147735}

Submitted on 4 Jun 2019

HAL is a multi-disciplinary open access archive for the deposit and dissemination of scientific research documents, whether they are published or not. The documents may come from teaching and research institutions in France or abroad, or from public or private research centers.
L'archive ouverte pluridisciplinaire HAL, est destinée au dépôt et à la diffusion de documents scientifiques de niveau recherche, publiés ou non, émanant des établissements d'enseignement et de recherche français ou étrangers, des laboratoires publics ou privés. 


\title{
On some $k$-scoring rules for committee elections: agreement and Condorcet Principle
}

\author{
Mostapha Diss · Eric Kamwa • Abdelmonaim Tlidi
}

17 May 2019

\begin{abstract}
Given a collection of individual preferences on a set of candidates and a desired number of winners, a multi-winner voting rule outputs groups of winners, which we call committees. In this paper, we consider five multi-winner voting rules widely studied in the literature of social choice theory: the $k$-Plurality rule, the $k$-Borda rule, the $k$-Negative Plurality rule, the Bloc rule, and the Chamberlin-Courant rule. The objective of this paper is to provide a comparison of these multi-winner voting rules according to many principles by taking into account a probabilistic approach using the well-known Impartial Anonymous Culture (IAC) assumption. We first evaluate the probability that each pair of the considered voting rules selects the same unique committee in order to identify which multi-winner rules are the most likely to agree for a given number of candidates and a fixed target size of the committee. In this matter, our results show that the Chamberlin-Courant rule and the $k$-Plurality rule on one side, and the $k$-Borda rule and the Bloc rule on the other side, are the pairs of rules that most agree in comparison to other pairs. Furthermore, we evaluate the probability of every multi-winner voting rule selecting the Condorcet committee à la Gehrlein when it exists. The Condorcet committee à la Gehrlein is a fixed-size committee such that every member defeats every non-member in
\end{abstract}

Mostapha Diss

Univ Lyon, UJM Saint-Etienne, CNRS, GATE L-SE UMR 5824, F-42023 Saint-Etienne, France. E-mail: diss@gate.cnrs.fr

Eric Kamwa

Université des Antilles, Faculté de Droit et d'Economie de la Martinique and Laboratoire Caribéen de Sciences Sociales LC2S UMR CNRS 8053, F-97275 Schoelcher Cedex, France.

E-mail: eric.kamwa@univ-antilles.fr

Abdelmonaim Tlidi

University Cadi Ayyad of Marrakesh, GREER, National School of Applied Science-Safi, Route Sidi Bouzid, B.P. 63, 4600o Safi, Morocco.

E-mail: mtlidi2010@gmail.com 
pairwise comparisons. In addition, we compare the considered multi-winner voting rules according to their ability (susceptibility) to select a committee containing the Condorcet winner (loser) when one exists. Here, our results tell us that in general, the $k$-Borda rule has the highest performance amongst all the considered voting rules. Finally, we highlight that this paper is one of the very rare contributions in the literature giving exact results under the Impartial Anonymous Culture (IAC) condition for the case of four candidates.

Keywords: Voting, Committee, Borda, Condorcet, Scoring rules, ChamberlinCourant.

JEL Classification Number: $\mathrm{D}_{71}, \mathrm{D}_{72}$

Acknowledgements: The authors would like to thank Vincent Merlin for his perceptive comments regarding a first draft of this paper. We are also indebted to Winfried Bruns and all the Normaliz Team for their useful discussions about the volume calculation of some polytopes using the software Normaliz. We further thank Shoaib Abdul Basit and Adrien Lutz for their help in proofreading the paper. Early drafts of this paper have been presented at the meeting of the Association of Southern European Economic Theorists in Florence (November 2018), the 14th meeting of the Society for Social Choice and Welfare in Seoul (June 2018), the CREM seminar in Caen Normandy university (December 2017), the LED seminar in Paris 8 university (January 2018), and the seminar of the department of Mathematics in Kenitra university (January 2018). The authors are grateful for comments received from the participants at all those seminars and conferences. The first author would like to acknowledge the financial support from Université de Lyon (project INDEPTH Scientific Breakthrough Program of IDEX Lyon) within the program Investissement d'Avenir (ANR-16-IDEX-0005). Note that a previous version of this paper has been circulated under the title "The Chamberlin-Courant Rule and the $k$-Scoring Rules: Agreement and Condorcet Committee Consistency".

\section{Introduction}

A multi-winner voting rule or a committee selection rule outputs groups of winners (candidates, alternatives, etc.) with a certain size, which we call committees, taking into account a collection of individual preferences, i.e., preference profiles, on a given set of candidates. Since the pioneer works of Dodgson (1884, 1876), Droop (1881), and Sterne (1871), the committee selection setting has been of great interest in the late 80 for many researchers including political scientists, economists, and computer scientists. This research subject is still of interest in the recent literature. For instance, Brams and Brill (2018), Brams 
et al. (2019), Brill et al. (2018), Elkind et al. (2017), Faliszewski et al. (2018), Kilgour (2018), Skowron et al. (2016) have recently examined the properties of some voting rules in multi-winner contexts and studied a set of natural properties against which these voting rules can be examined.

Obviously, the use of a given multi-winner voting rule must be consistent with a specific objective to be pursued. This objective may be to draw up a first list of candidates, i.e., shortlisting, that will be submitted afterwards to deliberation in order to determine a final winner from this list, or to select a given committee that accurately reflects the electorate. Consequently, as is the case in the single-winner framework, there are also many multi-winner voting rules based on different ideas and principles that have been designed for the purpose of electing a fixed number of winners among a set of available candidates.

One possibility is to use those voting rules which are built for the singlewinner setting using a scoring function, namely a function which associates with each preference profile and a candidate a real number to be interpreted as the score obtained by such a candidate for the considered preference profile. In other words, according to this family of rules, each candidate gets some points from each voter according to her position in the voter's preference, and in the end the candidate with the highest aggregated score is elected. Among these rules there are, for instance, the Borda rule, the Plurality rule and the Negative Plurality rule. Obviously, since the scores allow all the candidates to be ordered, the voting rule adapted to the multi-winner context selects, for every preference profile, those candidates having the $k$ greatest aggregated scores, where $k$ stands for the target size of the committee that we aim to select. The study of the features of multi-winner voting rules in this context has received increasing interest in the recent literature on social choice theory. Without being exhaustive, the following papers deal with this approach: Bock et al. (1998), Debord (1993), Diss and Doghmi (2016), Dummett (1984), Elkind et al. (2017), Faliszewski et al. (2011, 2016, 2018), Kamwa and Merlin (2015), Obraztsova et al. (2011), Skowron et al. (2016).

Another possibility that we might use is to build the choice from the pairwise comparisons in the spirit of the Condorcet Principle, which is perhaps the most widely accepted desiderata for social choice theorists: if there is a candidate (the Condorcet winner) who would defeat every other candidate in a pairwise race, then that candidate should be the winner of the election. First, we can take for instance the approach of Gehrlein (1985) where we want to select a committee such that any member wins against any committee nonmember in pairwise majority comparisons. This is known in the literature as the $k$-Condorcet set or the Condorcet committee à la Gehrlein. We might also be concerned about the possibility of selecting a subset of candidates such that 
no candidate in this subset can be defeated by any candidate from outside the subset on the basis of pairwise majority comparisons. This also comes from Gehrlein (1985) and is known in the literature as the weak k-Condorcet set à la Gehrlein (see for instance Barberà and Coelho, 2008). The reader will easily understand that these two concepts are simple generalizations of the wellknown concepts of the strong Condorcet winner and the weak Condorcet winner ${ }^{1}$ to the committee selection setting.

Obviously, there are many other contributions where various multiwinner systems are used with different types of individual preferences and votes. The reader may refer for instance to the works of Aziz et al. (2017), Barberà and Coelho (2008), Brams (2008), Brams et al. (2005, 2006), Elkind et al. (2011), Fishburn (1981), Kamwa (2017), Kamwa and Merlin (2018), Kaymak and Sanver (2003), Kilgour (2010), Kilgour and Marshall (2012), Ratliff (2003), among others.

Scholars have conducted many comparative studies of single-winner voting rules according to various principles. An overview of research in this matter will be provided later in the paper. In the present work, we extend this research to the multi-winner setting by comparing five well-known multiwinner rules which have attracted a considerable amount of attention in the recent literature of social choice theory. Several reasons justify the choice of our multi-winner voting rules. The common thread in all the rules that we focus on is that they are based on scoring functions. More exactly, we aim to compare the k-Plurality rule, the k-Borda rule, the k-Negative Plurality rule, the Bloc rule, and the Chamberlin-Courant rule (defined later), according to welldefined principles. These principles include (1) the ability of every pair of the considered multi-winner voting rules to select the same and unique committee of a given size; (2) the ability of every voting method to select a committee corresponding to the Condorcet committee à la Gehrlein when such a committee exists; (3) the ability of every rule to select the Condorcet winner among the chosen committee members in the class of elections for which the Condorcet winner exists; and (4) the sensitivity of every voting system to select the Condorcet loser when one exists. Recall that a Condorcet loser is a candidate who loses in a direct pairwise comparison to every other candidate. It is worthwhile noting that from the famous debate between Dodgson $(1884,1876)$ and the Society for Proportional Representation, it emerged that when electing committees, the Condorcet Principle cannot be totally ignored as it seems inconceivable to elect committees with dominated members. The

\footnotetext{
1 A strong Condorcet winner is a candidate that a majority of voters rank higher than every other candidate. A weak Condorcet winner is a candidate that no majority of voters rank below any other candidate. Throughout the paper, when we write Condorcet winner we mean strong Condorcet winner, unless we specifically write weak.
} 
present paper makes a contribution to this debate through the discussion of three items (2-4) which deal with Condorcet's idea.

We focus in this paper on elections with large electorates, where voters have linear orders on the candidates as their preference profiles. In addition, for technical reasons (described later) we only deal with elections where the number of candidates $m$ is included in the set $\{3,4,5,6\}$ with a target size $k$ of the committee defined such as $1 \leq k \leq m-1$. In order to deal with the comparison of the five multi-winner rules according to the four principles described above, our study requires information about how voters cast their votes. Notice that it is difficult to obtain enough information about voter preference profiles particularly in elections involving large electorates. As a consequence, in this paper we follow a theoretical approach by making a well-known assumption about how voters might behave in the election process. More exactly, all our comparisons are conducted using a probabilistic approach by assuming that the preference profiles of voters follow the widely used hypothesis of the Impartial Anonymous Culture (IAC). We emphasize that this paper is one of the rare contributions in the literature giving exact results under the Impartial Anonymous Culture (IAC) condition for the case of four candidates. To the best of our knowledge, the only papers in this category are: Bruns et al. (2019), Bubboloni et al. (2018), Diss and Doghmi (2016), and El Ouafdi et al. (2018).

Our first finding (item 1 ) is that, if the preference profiles of voters are allowed to meet the IAC condition, then the agreement between voting procedures is observed with non negligible probabilities. Our comparisons of the considered multi-winner rules according to the Condorcet Principle (items 24) show that in general the $k$-Borda rule displays superior performance in comparison to the other multi-winner rules. Moreover, we find that the $k$ Negative Plurality rule often exhibits extremely poor performance and is not a viable option for consideration on the basis of the considered Condorcet Principles.

The rest of the paper is structured as follows: Section 2 is devoted to basic notations, definitions as well as the methodology of calculations. Section 3 presents our results and Section 4 concludes the paper.

\section{Background notions}

2.1 Individual preferences

Let $N=\{1, \ldots, n\}$ be a set of $n \geq 3$ voters and $A=\{a, b, c, \ldots\}$ the set of $m \geq$ 3 candidates. We consider the framework in which each voter is assumed to 
have a linear order on the set of candidates from the most desirable candidate to the least desirable one. Notice that a linear order is a binary relation that is transitive, complete and antisymmetric. In addition, each voter is assumed to act according to her true preferences which clearly means that the setting of this paper does not support strategic voting. We denote by $\pi$ the preference profile of voters which identifies the specific linear ranking that each voter has on the candidates. In the sequel, we will simply write, $a b c \ldots$ to denote that candidate $a$ is ranked ahead of candidate $b$ who is ranked ahead of $c$ and so on. In this context, with $m$ candidates, there are $m$ ! possible strict rankings and a voting situation is defined by the vector $\tilde{n}=\left(n_{1}, \ldots, n_{t}, \ldots, n_{m !}\right)$ such that $\sum_{t=1}^{m !} n_{t}=n$. The notation $n_{t}$ refers to the number of voters endowed with each of the $m$ ! linear orders. For each pair of candidates $a, b \in A$, we denote by $n_{a b}$ the number of voters who rank candidate $a$ before candidate $b$. If $n_{a b}>n_{b a}$, we will say that candidate $a$ is majority preferred to candidate $b$ and we denote it by $a \mathbf{M} b$. A candidate is said to be a Condorcet winner if she wins in all of her pairwise elections. In other words, the Condorcet winner is $a$ when $a \mathbf{M} b$ for every candidate $b \neq a$ in the set of candidates $A$. Furthermore, a candidate is said to be a Condorcet loser if she is ranked below any other candidate by a majority of voters. In other words, $a$ is a Condorcet loser when $b \mathbf{M} a$ for every candidate $b \neq a$ in the set of candidates $A$. It is well known that a Condorcet winner and a Condorcet loser do not exist in every voting scenario. In addition, many voting rules are not Condorcet-consistent which means that, even when there is a Condorcet winner, such a candidate may lose and conversely, when there is a Condorcet loser, such a candidate may win.

As our framework is that of multi-winner elections, we denote by $k$ $(k<m)$ the number of candidates to be elected. In addition, we denote by $\mathfrak{C}(k, m)$ the set of all possible committees of size $k$ for $m$ candidates and we define $|\mathfrak{C}(k, m)|$ as the cardinality of this set. A committee $C \in \mathfrak{C}(k, m)$ is a Condorcet committee à la Gehrlein if each element in this committee beats each element outside it in terms of a pairwise majority comparison. Formally, this means that $\forall a \in C$ and $\forall b \in A \backslash C, a \mathbf{M} b$ is verified. As mentioned above, the Condorcet committee à la. Gehrlein has been suggested in order to avoid committees with dominated member(s). Nonetheless, the Condorcet committee $\grave{a}$ la Gehrlein does not always exist. That is the reason why other extensions of this concept have been suggested in the literature. The reader is referred to Barberà and Coelho (2008), Kamwa (2017), Ratliff (2003) for more informa- 
tion on these notions. ${ }^{2}$ Finally, it is important to stress that when a Condorcet committee à la Gehrlein exists, then it always includes the Condorcet winner and never contains the Condorcet loser.

\subsection{Multi-winner voting rules}

A multi-winner voting rule maps the preferences of all voters in $N$ to a subset of $A$, which consequently specifies the composition of the committee. A scoring rule is a voting system that gives points to candidates in accordance with the position they occupy in voters' rankings. The total number of points received by a candidate defines her score under the considered rule. Formally, a scoring rule can be expressed by a sequence of real numbers $s=\left(s_{1}, s_{2}, \ldots, s_{m}\right)$ such that $s_{1} \geq s_{2} \geq \cdots \geq s_{m}$ and $s_{1}>s_{m}$. Each of the $n$ voters ranks the candidates assigning $s_{1}$ points to the one ranked first, $s_{2}$ points to the one ranked second, and so on until $s_{m}$, the number of points assigned to the candidate ranked last. In single-winner elections, the winner is the candidate with the highest score summed over all voters. Naturally, in multi-winner elections, a subset of $k$ winners or the elected committee of size $k$ is composed by the $k$ candidates with the $k$ highest scores.

We examine five multi-winner voting rules. As mentioned before, the first reason for considering these voting rules is because they are based on the general scoring protocol. The second reason is because the study of the characteristics and properties of these multi-winner scoring methods has generated the most extensive body of research in the recent literature (e.g., Diss and Doghmi, 2016, Elkind et al., 2017, Faliszewski et al., 2011, 2016, 2018, Kamwa and Merlin, 2015, Obraztsova et al., 2011, Skowron et al., 2016) in comparison with other multi-winner voting rules. The multi-winner scoring rules considered in this paper are the following:

k-Plurality: Under the Plurality rule the vector of scores is given by $s=(1,0, \ldots, 0)$, i.e., the Plurality score of a candidate is the total number of voters who rank this candidate at the top of their rankings. Under the $k$-Plurality, the same vector is used and this rule returns the $k$ candidates with the $k$ highest Plurality scores. Notice that the $k$-Plurality rule is also called Single Non-transferable Vote (e.g., Elkind et al., 2017).

\footnotetext{
${ }^{2}$ Notice that the Condorcet committee à la Fishburn (Fishburn, 1981) has also been suggested in the literature in order to avoid dominated committees. In this framework, it is assumed that the voters have preferences over committees that satisfy certain conditions, and Fishburn (1981) defines a Condorcet committee as a committee that is preferred to every other committee by a majority of voters. Kamwa and Merlin (2018), Kaymak and Sanver (2003) have tried to bridge the two concepts through preference extensions. Finally, we should mention that other approaches are also taken into consideration by Elkind et al. (2015), Ratliff (2006) and Brams et al. (2006) in order to compare sets of candidates.
} 
k-Borda: This rule selects the $k$ candidates with the $k$ highest Borda scores. The Borda rule gives $m-j$ points to a candidate each time she is ranked $j$-th, i.e., $s=(m-1, m-2, \ldots, 1,0)$, and the Borda score of a candidate is the sum of the points received.

k-Negative Plurality: Also called the $k$-Antiplurality rule, this rule returns the $k$ candidates with the $k$ lowest number of last places in the voters' rankings. In other words, the vector of score that is used here corresponds to $s=(1,1, \ldots, 1,0)$.

Bloc: This rule returns the $k$ candidates with the highest $k$-approval scores. The $k$-approval score of a candidate is equal to the number of voters who rank this candidate among their $k$ top ranked candidates, i.e., $s=(\underbrace{1, \ldots, 1}_{k}, 0, \ldots, 0)$. Notice first that Bloc is equivalent to $k$-Plurality and $k$-Negative Plurality when $k=1$ and $k=m-1$, respectively. Furthermore, notice that the Bloc rule is also called Limited Voting (e.g., Kamwa and Merlin, 2015) or Constant Scoring Rule (e.g., Gehrlein, 1985).

Chamberlin-Courant: Under this rule we fix a scoring vector of length $m$, and each voter's score for a given committee is defined as the score that she assigns to her most preferred candidate in that committee; the goal is then to find a committee that maximizes the joint scores of all voters. Notice that in line with Chamberlin and Courant (1983), in this paper we consider the most often used vector of scores which is the one defined by Borda, i.e., $s=(m-1, m-2, \ldots, 1,0)$. Formally, let $r_{i x}$ be the rank of candidate $x$ in voter $i$ 's ranking and $w\left(r_{i x}\right)=m-r_{i x}$ the corresponding Borda weight. We denote by $N_{x}(C, \pi)$ the set of voters for which the representative in committee $C$ is candidate $x$ for profile $\pi$. In other words, for the corresponding profile $\pi$, candidate $x$ is the most preferred in the committee $C$ for all voters in $N_{x}(C, \pi)$. According to Chamberlin and Courant (1983), the ChamberlinCourant rule selects the committee which maximizes the representativeness value:

$$
\alpha(C, \pi)=\sum_{x \in C} \sum_{i \in N_{x}(C, \pi)} w\left(r_{i x}\right)
$$

The Chamberlin-Courant rule is equivalent to the Borda rule for $k=1$ (Chamberlin and Courant, 1983) and to the $k$-Plurality rule for $k=m-1$ (Kamwa and Merlin, 2014). It is important to stress here that this paper only deals with the utilitarian approach of the Chamberlin-Courant rule which is described in (1). Besides this approach, there are other ways of calculating the representativeness value. The reader is referred to Betzler et al. (2013) who 
suggested the egalitarian variants of the Chamberlin-Courant rule. For more details on the other variant and the links between both versions, the reader is referred to the papers of Betzler et al. (2013), Elkind et al. (2017), Procaccia et al. (2008), Skowron et al. (2013a, 2015). Notice that both the utilitarian and the egalitarian approaches are very hard to manage (Elkind et al., 2017). In other words, finding the outcome(s) under the Chamberlin-Courant rule can be computationally intractable particularly when the number of candidates and the size of the committee to be elected increase. This drawback may compromise the use of this rule in real elections with a huge number of candidates, to the benefit of alternative multi-winner voting rules. As a consequence, $\mathrm{Lu}$ and Boutilier (2011) have proposed some algorithms for approximating the Chamberlin-Courant rule. For their own purposes, Potthoff and Brams (1998) showed that integer programming can be used in order to determine the winners under the Chamberlin-Courant rule. Nevertheless, as pointed out by Procaccia et al. (2008), the integer programming does not overcome the complexity of the Chamberlin-Courant rule.

In order to illustrate the different notions presented above, we provide the following example.

Example 1. Consider a profile $\pi$ with $n=22$ individuals voting on the set $A=$ $\{a, b, c, d\}$ of $m=4$ candidates. The preference of every individual $i$ is described as follows:

\begin{tabular}{|l|c|c|r|}
\hline Voters & $i=1, \ldots, 4$ & $i=5, \ldots, 8$ & $i=9, \ldots, 12$ \\
\hline Rankings & $a b c d$ & $a d c b$ & $c b d a$ \\
\hline \multicolumn{3}{|c|}{$|c| c \mid$} \\
\hline Voters & $i=13, \ldots, 16$ & $i=17, \ldots, 22$ \\
\hline Rankings & $b c d a$ & $d b c a$ \\
\hline
\end{tabular}

The scores of the candidates under the Plurality rule, the Negative Plurality rule, the Borda rule, and the Bloc rule (with $k=2$ ) are calculated as follows:

\begin{tabular}{|l|c|c|c|c|}
\cline { 2 - 5 } \multicolumn{1}{c|}{} & $a$ & $b$ & $c$ & $d$ \\
\hline The Plurality rule & 8 & 4 & 4 & 6 \\
\hline The Negative Plurality rule & 8 & 18 & 22 & 18 \\
\hline The Borda rule & 24 & 40 & 34 & 34 \\
\hline The Bloc rule $(k=2)$ & 8 & 18 & 8 & 10 \\
\hline
\end{tabular}

After all computations assuming that $k=2$, the scores of the $6=\mid(\mathfrak{C}(k=2, m=4) \mid$ possible committees under the Chamberlin-Courant rule are: $\alpha(\{a, b\}, \pi)=56$ $; \alpha(\{a, c\}, \pi)=\alpha(\{a, d\}, \pi)=\alpha(\{c, d\}, \pi)=50 ; \alpha(\{b, d\}, \pi)=54$; $\alpha(\{b, c\}, \pi)=48$. In other words, if the target size of the elected committee is $k=2$, the Chamberlin-Courant rule selects $\{a, b\}$ whereas the 2-Plurality rule chooses $\{a, d\}$; the committees $\{b, c\}$ and $\{c, d\}$ tie for the 2-Negative Plurality; 
the committees $\{b, c\}$ and $\{b, d\}$ tie for the 2-Borda rule; finally, the Bloc rule selects the committee $\{b, d\}$. Notice that $\{b, c\}$ is the Condorcet committee à la Gehrlein because $b \mathbf{M} a, b \mathbf{M} d, c \mathbf{M} a$, and $c \mathbf{M} d$. Furthermore, candidate $b$ is the Condorcet winner because $b \mathbf{M} a, b \mathbf{M} c$, and $b \mathbf{M} d$, whereas candidate $d$ is the Condorcet loser because $a \mathbf{M} d, b \mathbf{M} d$, and $c \mathbf{M} d$.

\subsection{Methodology}

Before giving our results, we describe the methodology applied in order to calculate our probabilities. To achieve this goal, we assume that all voting situations $\tilde{n}=\left(n_{1}, \ldots, n_{t}, \ldots, n_{m !}\right)$ with $n=\sum_{t=1}^{m !} n_{t}$ for specified $n$ and $m$ are equally likely to be observed. This is called in the literature the Impartial Anonymous Culture (IAC) condition. Introduced by Gehrlein and Fishburn (1976) in social choice literature, the IAC assumption is one of the most widely used assumptions in the literature when computing the likelihood of voting events. For further details on the IAC and other assumptions, we refer the reader to Gehrlein and Lepelley $(2017,2011)$.

Obtaining the probability of an electoral event under the IAC assumption is accomplished by the computation of two elements. The first one is the total number of voting situations $\tilde{n}=\left(n_{1}, \ldots, n_{t}, \ldots, n_{m !}\right)$; it is well known that for $n$ voters and $m$ candidates the total number of voting situations $\tilde{n}$ is explicitly given by the binomial coefficient $\left(\begin{array}{c}n+m !-1 \\ m !-1\end{array}\right)$. The second element that has to be calculated is the number of voting situations associated with the corresponding electoral event that we need to evaluate, which can be reduced to a finite system of linear constraints with rational coefficients. Furthermore, it is well known that the calculations of the limiting probability, as the number of voters tends to infinity, under the IAC condition, are simply reduced to the computation of volumes of convex polytopes. In other words, for our purpose in this paper, every probability requires the calculation of two volumes of certain polytopes of dimension $m$ !. For this, our volumes are found with the use of the algorithm Convex which is a MAPLE package for convex geometry written by Franz (2016). This package works with the same general procedure that was implemented in Cervone et al. (2005) and recently used in other studies, e.g., Diss and Doghmi (2016), Diss and Gehrlein (2015, 2012), Gehrlein et al. (2015) and Moyouwou and Tchantcho (2017). This technique is used in this paper in order to obtain all our exact results for $m=3$ and $m=4$, except for the probabilities displayed in Table 11 for which we used Normaliz (Bruns et al., 2017, 2019) which works with the same procedure as Convex. However, the two methods are not efficient when, as in this paper, the number of candidates is equal to 5 and 6 . Unfortunately, the computation times with four candidates range from seconds to a few days or even weeks on a 
fast machine, particularly for the calculations involving the Borda score (i.e., under the $k$-Borda and the Chamberlin-Courant rules). In other words, our calculations for $m=3$ and $m=4$ cannot be expanded to $m=5$ and $m=6$ since the computations need relatively high memory when the number of candidates increases, and consequently our computer hardware does not allow the calculation of the volumes associated with these cases in a reasonable time. ${ }^{3}$

For this reason, computer simulations are used to evaluate our probabilities for $m=5$ and $m=6$. We describe in the following the applied methodology. Let us consider as an example the probability for which the ChamberlinCourant rule and the $k$-Plurality rule both select the same unique committee for a specific pair $(k, m)$. In the beginning, we randomly generate a voting situation of length $m$ ! with a number of voters $n$ tending to infinity. For this purpose, we consider a number of voters equal to 100,000. In the second step, we check whether the conditions for which the Chamberlin-Courant rule and the $k$-Plurality rule agree on the same and unique committee are fulfilled or not. These two steps are iterated 1,000,000 times to obtain the number of voting situations for which the Chamberlin-Courant rule and the $k$-Plurality rule agree. Finally, the probability of agreement is calculated as the quotient of the number previously obtained in the latest step over the total number of simulated voting situations, i.e., 1,000,000. Notice that, we can also generate simulations with more than 100, 000 voters and more than 1,000,000 iterations. We have ignored this option since small differences are observed between our probabilities and those obtained with high parameters.

We provide in the Appendix some codes to illustrate how the different probabilities given in this paper are calculated. The detail of the other codes are available upon simple request from the authors.

3 The only volumes that we can provide are the ones describing the region which includes all the voting situations $\tilde{n}=\left(n_{1}, \ldots, n_{t}, \ldots, n_{m !}\right)$. Indeed, any voting situation $\tilde{n}$ can be rewritten as $\tilde{p}=\left(p_{1}, \ldots, p_{t}, \ldots, p_{m !}\right)$ such that $\sum_{t=1}^{m !} p_{t}=1$ and $p_{t}=\frac{n_{t}}{n} \geq 0$ denoting the proportion of the $n$ voters with the associated $t^{\text {th }}$ linear ordering in a given election with large electorates. Then, the set of all voting situations $\tilde{p}$ corresponds to an $(m !-1)$-dimensional simplex. Observing that the edges of the simplex are all equal to $\sqrt{2}$ and using known geometric formulas, the volume of the IAC region for $m$ candidates is given by:

$$
\operatorname{Volume}(I A C, m)=\frac{\sqrt{m !}}{(m !-1) !}=\frac{m !^{3 / 2}}{m ! !} .
$$




\section{Results}

3.1 The selection of the same unique committee

The question of how different the outcomes of two voting rules can be when applied on the same preference profile has been widely answered in the literature when the aim is to select a single winner from the preferences of an entire population. In this literature, earlier studies teach us that the existence of the Condorcet winner indirectly reveals something about the discrepancies of voting procedures. In order to achieve this end, many studies in the literature have focused on the likelihood that different voting procedures tend to always elect the Condorcet winner when such a candidate exists. For instance, Gehrlein (1999) examines the likelihood that scoring rules and scoring elimination rules ${ }^{4}$ tend to always elect the Condorcet winner in three-candidate elections. For more results, we also refer the reader to Fishburn (1977), Gehrlein and Fishburn (1978a), Klamler (2004, 2003), Mathur and Bhattacharyya (2017), Merlin et al. (2000), Ratliff (2002, 2001), Regenwetter and Grofman (1998), who also analyze the question of selecting the same winner (Condorcet winner) under various voting rules. Other studies analyze the choices in contexts where there is no Condorcet winner (e.g., Merlin et al., 2000, Nurmi, 1988, 1987). These studies mainly teach us that the agreement between different voting rules is not insignificant.

Our first contribution in this paper is then to extend the literature analyzing the agreement between voting procedures to the multi-winner context. We will particularly investigate the situation where each pair of the considered multi-winner voting rules selects the same unique committee. For this purpose, we explore the cases for $m=3,4,5,6$ and all possible values of $k$ with a number of voters tending to infinity. Our first finding is that, if the preference profiles of voters are allowed to follow the IAC condition, then even in the multi-winner setting the agreement between voting procedures is observed with non-negligible probability.

As stated above, the Chamberlin-Courant rule is equivalent to the Borda rule for $k=1$ and to the $k$-Plurality rule for $k=m-1$. In addition, the Bloc rule is equivalent to the $k$-Plurality rule and the $k$-Negative Plurality rule when $k=1$ and $k=m-1$, respectively. To our knowledge, except for these values, there are no other values of $k$ and $m$ such that a pair of the considered multi-winner voting rules always agrees on the same unique committee. Consequently, our first objective in this section is to find how

4 Scoring elimination rules, also called multistage elimination scoring rules, give points to candidates according to their rank in voters' preference orders and then eliminate the candidate(s) with the lowest number of points. 
often the outcome of a given pair of the considered multi-winner voting rules coincides on the same unique committee for any pair $(k, m)$. The symmetry of IAC with respect to candidates requires that the probability of only one case has to be found and then we multiply this result by the number of possible committees given by $|\mathfrak{C}(k=2, m=4)|$ in order to find the total probability of agreement. For example, with $m=4$ and $k=2$, we first calculate the probability that two given multi-winner voting rules both select the same unique committee $\{a, b\}$ and then we multiply this probability by $6=\mid \mathfrak{C}(k=$ $2, m=4) \mid$, to take into account the fact that other possible committees than $\{a, b\}$ can also be the winner under the two voting rules.

The results of our computations are provided in Tables (1) to (10). Several lessons may be drawn from these probabilities. First, for a given $m$, we observe that the probability of the agreement between any pair of multi-winner voting rules exhibits the same behavior, i.e., it first decreases and then increases as $k$ increases. This means that the probability of the agreement is significantly high when the target size of the committee $k$ is sufficiently small (around 1) or sufficiently high (around $m-1$ ). Our intuition regarding this remark is related to the fact that the value of $|\mathfrak{C}(k, m)|$ is small ${ }^{5}$ when $k$ is close to 1 or $m-1$, which makes the agreement more probable for these values of $k$ than the other values. Second, it is quite natural that for a given size $k$ of the committee the probability of the agreement tends to decrease as the number of candidates $m$ increases. This fact generally holds under each pair of rules. For instance, with $k=2$, the probability of agreement between the Chamberlin-Courant rule and the $k$-Plurality rule decreases from 0.7223 for $m=4$ to 0.5232 for $m=5$ and then to 0.3868 for $m=6$. Third, we highlight the fact that the ChamberlinCourant rule and the $k$-Plurality rule on one side and the $k$-Borda rule and the Bloc rule on the other side are the pairs of rules that agree the most in comparison to other pairs. More precisely, the $k$-Plurality rule tends in sum to dominate the other rules in terms of the agreement with the ChamberlinCourant rule whereas the $k$-Negative Plurality rule performs the worst; on the other hand, the $k$-Borda rule tends to dominate the other rules in terms of its agreement with the Bloc rule whereas the $k$-Plurality appears to exhibit a much poorer performance. Finally, our probabilities show that the agreement that we investigate in this paper is observed with non-negligible probability for any pair of multi-winner voting rules. In other words, we want to highlight in this section the fact that many multi-winner voting rules are quite similar, particularly for some values of $m$ and $k$.

It would have been interesting to find the probability of other types of agreements. Indeed, in addition to our probability (say $P r_{1}$ ), a second possibility (say $P r_{2}$ ) could be the probability for which a first multi-winner voting

\footnotetext{
5 For instance, $|\mathfrak{C}(k=1, m=5)|=|\mathfrak{C}(k=4, m=5)|=5$ and $|\mathfrak{C}(k=2, m=5)|=\mid \mathfrak{C}(k=$ $3, m=5) \mid=10$.
} 
rule selects only one committee and that this committee is included in the outcome set of a second multi-winner voting rule that may contain more than one committee. It is obvious that for any pair of rules, $P r_{2}$ is equal to $P r_{1}$ plus the probability of the cases for which the first rule selects only one committee (e.g., $\{a, b\})$ and that the second rule chooses two or more committees (e.g., $\{a, b\}$ and $\{b, c\}$ ). A third possibility (say $\operatorname{Pr}_{3}$ ) could be to evaluate for any pair of rules the probability under which the first rule selects one or more committees and that these committees are included in the outcome set of the second rule. In other words, the set of winning committees under a first rule is included in the one of a second rule. Again, it is obvious that $\mathrm{Pr}_{3}$ is equal to $\mathrm{Pr}_{2}$ plus the probability of the cases for which the first rule selects two of more committees (e.g., $\{a, b\}$ and $\{b, c\}$ ) and that the second rule chooses the committees selected by the first rule plus (or not) other possible committees with the same size (e.g., $\{a, b\},\{b, c\}$, and $\{a, c\}$ ). Obviously, the probability $\mathrm{Pr}_{1}$ analyses the cases where the rules are decisive (also called resolute); the probability $\mathrm{Pr}_{2}$ assumes that only one rule is decisive whereas the second rule is not; and $\mathrm{Pr}_{3}$ supposes that the two considered rules are not decisive. It could be relevant to distinguish the cases where the rules are decisive from those where they are not, because breaking ties may lead to more complex problems: the choice of the tie-breaking rule is not entirely neutral because it can be used for strategic manipulation purposes as recently pointed out by Aziz al. (2013), Mattei et al. (2014) and Obraztsova et al. (2011). However, as the size of the electorate tends to be large, which is our concern in this paper, the result $P r_{1}=P r_{2}=P r_{3}$ holds. Why? To obtain $P r_{1}$, for instance, our polytopes are only described by strict inequalities, whereas under $\mathrm{Pr}_{2}$ and $\mathrm{Pr}_{3}$ we need weak inequalities. Furthermore, we know that when $n$ increases significantly, these weak inequalities fall to make room for strict inequalities and we recover $P r_{1}$. As noticed above, the calculations of the limiting probability under the IAC condition are simply reduced to computations of volumes of convex polytopes. Discounting ties has no impact because the volume of a region is the same regardless of whether the region includes its bounding hyperplanes or not, and it is exactly the points lying on these bounding hyperplanes that correspond to the ties (included in the voting situations of $\mathrm{Pr}_{2}$ and $\mathrm{Pr}_{3}$ but not in $\left.P r_{1}\right)$. For more details, we refer the reader to footnote (3) in Cervone et al. (2005).

Let us also admit that apart from $P r_{1}$ (and eventually $P r_{2}$ and $P r_{3}$ ), other interesting types of probabilities can be considered. As one can see, $P r_{1}$ addresses the agreement by only assuming an overlap of the outcome sets that focuses on the committees as a whole. One can go further and imagine the agreement in terms of members; in other words, one would determine the probability that a given candidate elected with a first rule will also be chosen by a second rule with (or without) the same value of $k$. It could be worth 
exploring the agreement in this way but the discussion of this case and other possibilities of agreement is beyond the scope of this section.

Table 1: Agreement between Chamberlin-Courant and $k$ Plurality

\begin{tabular}{|c|cccc|}
\hline $\begin{array}{c}m \rightarrow \\
k \downarrow\end{array}$ & 3 & 4 & 5 & 6 \\
\hline 1 & 0.8241 & 0.6775 & 0.5668 & 0.4812 \\
2 & 1 & 0.7223 & 0.5232 & 0.3868 \\
3 & -- & 1 & 0.6503 & 0.4355 \\
4 & -- & -- & 1 & 0.6050 \\
5 & -- & -- & -- & 1 \\
\hline
\end{tabular}

Table 3: Agreement between Chamberlin-Courant and Bloc

\begin{tabular}{|c|cccc|}
\hline $\begin{array}{c}m \rightarrow \\
k \downarrow\end{array}$ & 3 & 4 & 5 & 6 \\
\hline 1 & 0.8241 & 0.6775 & 0.5668 & 0.4812 \\
2 & 0.5231 & 0.4537 & 0.4665 & 0.4435 \\
3 & -- & 0.3754 & 0.2525 & 0.2271 \\
4 & -- & -- & 0.2926 & 0.1566 \\
5 & -- & -- & -- & 0.2387 \\
\hline
\end{tabular}

Table 5: Agreement between $k$ Plurality and $k$-Borda

\begin{tabular}{|c|cccc|}
\hline $\begin{array}{c}m \rightarrow \\
k \downarrow\end{array}$ & 3 & 4 & 5 & 6 \\
\hline 1 & 0.8241 & 0.6775 & 0.5668 & 0.4812 \\
2 & 0.6872 & 0.5397 & 0.4124 & 0.3148 \\
3 & -- & 0.5829 & 0.3953 & 0.2705 \\
4 & -- & -- & 0.5155 & 0.3050 \\
5 & -- & -- & -- & 0.4594 \\
\hline
\end{tabular}

Table 2: Agreement between Chamberlin-Courant and $k$-Borda

\begin{tabular}{|c|cccc|}
\hline $\begin{array}{c}m \rightarrow \\
k \downarrow\end{array}$ & 3 & 4 & 5 & 6 \\
\hline 1 & 1 & 1 & 1 & 1 \\
2 & 0.6872 & 0.5732 & 0.5064 & 0.4713 \\
3 & -- & 0.5829 & 0.4181 & 0.3282 \\
4 & -- & -- & 0.5155 & 0.3229 \\
5 & -- & -- & -- & 0.4594 \\
\hline
\end{tabular}

Table 4: Agreement between Chamberlin-Courant and $k$ Negative Plurality

\begin{tabular}{|c|cccc|}
\hline $\begin{array}{c}m \rightarrow \\
k \downarrow\end{array}$ & 3 & 4 & 5 & 6 \\
\hline 1 & 0.6872 & 0.5829 & 0.5143 & 0.4573 \\
2 & 0.5231 & 0.3173 & 0.2172 & 0.1593 \\
3 & -- & 0.3754 & 0.1806 & 0.1031 \\
4 & -- & -- & 0.2926 & 0.1172 \\
5 & -- & -- & -- & 0.2387 \\
\hline
\end{tabular}

Table 6: Agreement between $k$ Plurality and Bloc

\begin{tabular}{|c|cccc|}
\hline $\begin{array}{c}m \rightarrow \\
k \downarrow\end{array}$ & 3 & 4 & 5 & 6 \\
\hline 1 & 1 & 1 & 1 & 1 \\
2 & 0.5231 & 0.3967 & 0.3374 & 0.2962 \\
3 & -- & 0.3754 & 0.2297 & 0.1610 \\
4 & -- & -- & 0.2926 & 0.1447 \\
5 & -- & -- & -- & 0.2387 \\
\hline
\end{tabular}


Table 7: Agreement between $k$ Plurality and $k$-Negative Plurality

\begin{tabular}{|c|cccc|}
\hline $\begin{array}{c}m \rightarrow \\
k \downarrow\end{array}$ & 3 & 4 & 5 & 6 \\
\hline 1 & 0.5231 & 0.3754 & 0.2937 & 0.2361 \\
2 & 0.5231 & 0.2839 & 0.1704 & 0.1093 \\
3 & -- & 0.3754 & 0.1677 & 0.0878 \\
4 & -- & -- & 0.2926 & 0.1120 \\
5 & -- & -- & -- & 0.2387 \\
\hline
\end{tabular}

Table 9: Agreement between $k$ Borda and $k$-Negative Plurality

\begin{tabular}{|c|cccc|}
\hline $\begin{array}{c}m \rightarrow \\
k \downarrow\end{array}$ & 3 & 4 & 5 & 6 \\
\hline 1 & 0.6872 & 0.5829 & 0.5143 & 0.4573 \\
2 & 0.8241 & 0.5397 & 0.3938 & 0.3050 \\
3 & -- & 0.7368 & 0.4108 & 0.2751 \\
4 & -- & -- & 0.5648 & 0.3152 \\
5 & -- & -- & -- & 0.4818 \\
\hline
\end{tabular}

Table 8: Agreement between $k$ Borda and Bloc

\begin{tabular}{|c|cccc|}
\hline $\begin{array}{c}m \rightarrow \\
k \downarrow\end{array}$ & 3 & 4 & 5 & 6 \\
\hline 1 & 0.8241 & 0.6775 & 0.5668 & 0.4812 \\
2 & 0.8241 & 0.6944 & 0.5768 & 0.4769 \\
3 & -- & 0.7368 & 0.5781 & 0.5060 \\
4 & -- & -- & 0.5648 & 0.4755 \\
5 & -- & -- & -- & 0.4818 \\
\hline
\end{tabular}

Table 10: Agreement between Bloc and $k$-Negative Plurality

\begin{tabular}{|c|cccc|}
\hline $\begin{array}{c}m \rightarrow \\
k \downarrow\end{array}$ & 3 & 4 & 5 & 6 \\
\hline 1 & 0.5231 & 0.3754 & 0.2937 & 0.2361 \\
2 & 1 & 0.3967 & 0.2285 & 0.1420 \\
3 & -- & 1 & 0.3334 & 0.1645 \\
4 & -- & -- & 1 & 0.2986 \\
5 & -- & -- & -- & 1 \\
\hline
\end{tabular}

The previous results showed that any pair of the different multi-winner voting rules we presented may end with the same results for many voting situations. In this section, we focus on the probability for which all these multi-winner voting methods agree for a given pair $(k, m)$. We focus again on the case of large electorates. Our results are displayed in Table (11). We can make some major observations based upon this table. The first remark is that the probabilities follow the same behavior observed in Tables (1) to (10) when we increase the number of candidates $m$ or the size of the committee $k$. In other words, when the number of candidates increases and the size of the committee remains constant then the probability of the agreement decreases. In addition, if we increase the size of the committee while maintaining a constant number of candidates, the probability of the agreement will first increase and then it will decrease. The second remark is that our results show that the probability of the agreement is not negligible particularly when the size of the committee $k$ is equal to 1 or $m-1$. There is a significant chance of an agreement occurring between the different multi-winner voting rules we presented; while this probability is around $52 \%$ for $m=3$, the probability of the agreement is expected to reach $25 \%$ for $m=5$ and $20 \%$ for $m=6$ when $k$ is equal to 1 or $m-1$, respectively. We arrive at our final observation: the probability of the agreement is the same for $k=1$ and $k=m-1$ regardless of the number of candidates. 
Table 11: Agreement between all the considered multi-winner voting rules

\begin{tabular}{|c|cccc|}
\hline $\begin{array}{c}m \rightarrow \\
k \downarrow\end{array}$ & 3 & 4 & 5 & 6 \\
\hline 1 & 0.5231 & 0.3550 & 0.2585 & 0.1930 \\
2 & 0.5231 & 0.1503 & 0.0685 & 0.0341 \\
3 & -- & 0.3550 & 0.0637 & 0.0198 \\
4 & -- & -- & 0.2568 & 0.0333 \\
5 & -- & -- & -- & 0.1942 \\
\hline
\end{tabular}

3.2 The selection of the Condorcet committee à la Gehrlein when it exists

We continue here the comparison between the multi-winner voting rules that we consider in this paper by evaluating the probability for each rule that it will select the Condorcet committee à la Gehrlein when it exists. In other words, we aim to analyse the Condorcet committee efficiency of each multiwinner voting rule. We focus again here on the case of large electorates and a number of candidates $m$ in the set $\{3,4,5,6\}$.

Notice that there is a large literature in social choice theory devoted exclusively to the Condorcet efficiency of single-winner voting rules. The Condorcet efficiency of a voting procedure is defined as the conditional probability that it will elect the Condorcet winner, given that a Condorcet winner exists. A detailed survey of early research on the Condorcet efficiency of voting rules can in particular be found in the recent books by Gehrlein and Lepelley (2017, 2011). However, it is striking how little attention has been paid to the Condorcet committee efficiency of multi-winner voting rules. To our knowledge, the only papers concerned with such a question are Gehrlein (1985) and Diss and Doghmi (2016).

We denote respectively by $C C E_{I A C \infty}^{k-P}(k, m), \quad C C E_{I A C \infty}^{k-N P}(k, m)$, $C C E_{I A C \infty}^{k-B}(k, m), \quad C C E_{I A C \infty}^{B}(k, m)$ and $C_{C C} E_{I A C \infty}^{C C R}(k, m)$ the limiting Condorcet committee efficiency of the $k$-Plurality rule, the $k$-Negative Plurality rule, the $k$-Borda rule, the Bloc rule, and the Chamberlin-Courant rule under the IAC condition as $n \rightarrow \infty$. Tables (12) to (16) provide our results. Notice that Tables (13) to (16) are drawn from Diss and Doghmi (2016). In other words, our findings in this section are set down in Table (12) which provides the Condorcet committee efficiency of the Chamberlin-Courant rule for various values of $m$ and $k$.

First, it is obvious from these tables that, for a given $k$, the Condorcet committee efficiency falls as $m$ rises. Moreover, for a given $m$, we observe that the Condorcet committee efficiency of the Chamberlin-Courant rule, the 
$k$-Negative Plurality rule, the $k$-Plurality rule, and the $k$-Borda rule exhibit the same behaviour, i.e., it first decreases and then increases as the target size of the committee $k$ increases. For instance, for $m=5$, the Condorcet committee efficiency of the Chamberlin-Courant rule varies from 0.8537 for $k=1$ to 0.4378 for $k=3$ and then it reaches 0.5106 for $k=4$; for $m=6$ this probability varies from 0.8458 for $k=1$ to 0.3574 for $k=4$ and then it reaches 0.4731 for $k=5$. However, this conclusion is not the same for the Bloc rule since its Condorcet committee efficiency first increases and then decreases as $k$ increases.

Second, as already noticed by Diss and Doghmi (2016), the $k$-Borda rule tends to perform better than the other $k$-scoring rules with more than an $80 \%$ Condorcet efficiency rate; it is followed by the Bloc rule. The $k$-Plurality rule performs the worst. Comparing our results to those of Diss and Doghmi (2016), it comes out that the Chamberlin-Courant rule does not perform better than the $k$-Borda rule and the Bloc rule (except for $k=1$ ); however, it performs better than the $k$-Plurality rule. We notice that for $k \leq\left\lfloor\frac{m}{2}\right\rfloor$, the ChamberlinCourant rule performs better than the $k$-Negative Plurality rule and for $k>$ $\left\lfloor\frac{m}{2}\right\rfloor$ we get the reverse, where \lfloor\rfloor stands for the integer part of the number. To summarize, our findings on Condorcet committee efficiency tell us that this rule can lead, most of the time, to committees with dominated members than the $k$-Borda rule and the Bloc rule.

Third, we recall some relationships that were developed in Gehrlein (1985) under the Impartial Culture (IC) ${ }^{6}$ assumption and then generalized to IAC in Diss and Doghmi (2016). In particular, $C C E_{I A C_{\infty}}^{k-P}(k, m)$ is the same as $C C E_{I A C_{\infty}}^{k-N P}(m-k, m)$. Moreover, for Bloc, it is found that $C C E_{I A C_{\infty}}^{B}(k, m)=$ $C C E_{I A C_{\infty}}^{B}(m-k, m)$ and $C C E_{I A C_{\infty}}^{k-B}(k, m)=C C E_{I A C_{\infty}}^{k-B}(m-k, m)$. Those relationships are not only true for $m=4$ candidates but also for $m=5$ and $m=6$. The small differences that we find for $m=5$ and $m=6$ are due to the fact that our results here are not exact but they are obtained using computer simulations, i.e., it is just due to the fact that the calculations are approximations. Consequently, the results in Tables (12) to (16) contain some information that allows us to check the margin of error of our simulation method for $m=5$ and $m=$ 6. More exactly, our results indicate a maximum error of about 0.0138 , which corresponds to $C C E_{I A C_{\infty}}^{k-B}(4,6)-C C E_{I A C_{\infty}}^{k-B}(2,6)=0.8372-0.8234=0.0138$.

\footnotetext{
${ }^{6}$ IC is also a well-known model in the literature of Social Choice Theory (Guilbaud, 1952). This condition considers the set of all preference profiles as a sample space. Recall that a voter preference profile is a collection of individual preferences expressed for every individual taking part in the vote. When strict preferences over the set of $m$ candidates are assumed, the IC assumption assumes that each voter is equally likely to pick any of the $m$ ! preferences. Notice that individual voter's preferences are not anonymous under the IC condition while they are under the IAC assumption. In addition, in the IC model, the votes are totally independent whereas under IAC the votes are correlated in a specific way. So, moving from IC to IAC allows us to evaluate the impact of introducing some degree of homogeneity in voters' preferences on the probability of paradoxes.
} 
Table 12: $C C E_{I A C_{\infty}}^{C C R}(k, m)$

\begin{tabular}{|c|cccc|}
\hline$m \rightarrow$ & 3 & 4 & 5 & 6 \\
$k \downarrow$ & & & & \\
\hline 1 & 0.9111 & 0.8706 & 0.8580 & 0.8457 \\
2 & 0.6296 & 0.5642 & 0.5398 & 0.5182 \\
3 & -- & 0.5516 & 0.4378 & 0.3757 \\
4 & -- & -- & 0.5100 & 0.3574 \\
5 & -- & -- & -- & 0.4701 \\
\hline
\end{tabular}

Table 14: $C C E_{I A C_{\infty}}^{k-N P}(k, m)$

\begin{tabular}{|c|cccc|}
\hline $\begin{array}{c}m \rightarrow \\
k \downarrow\end{array}$ & 3 & 4 & 5 & 6 \\
\hline 1 & 0.6296 & 0.5516 & 0.5104 & 0.4696 \\
2 & 0.8815 & 0.5427 & 0.4267 & 0.3529 \\
3 & -- & 0.7426 & 0.4521 & 0.3211 \\
4 & -- & -- & 0.6140 & 0.3642 \\
5 & -- & -- & -- & 0.5183 \\
\hline
\end{tabular}

Table 13: $C C E_{I A C_{\infty}}^{k-P}(k, m)$

\begin{tabular}{|c|cccc|}
\hline $\begin{array}{c}m \rightarrow \\
k \downarrow\end{array}$ & 3 & 4 & 5 & 6 \\
\hline 1 & 0.8815 & 0.7426 & 0.6143 & 0.5207 \\
2 & 0.6296 & 0.5427 & 0.4476 & 0.3638 \\
3 & -- & 0.5516 & 0.4199 & 0.3380 \\
4 & -- & -- & 0.5100 & 0.3322 \\
5 & -- & -- & -- & 0.4701 \\
\hline
\end{tabular}

Table 15: $C C E_{I A C_{\infty}}^{k-B}(k, m)$

\begin{tabular}{|c|cccc|}
\hline $\begin{array}{c}m \rightarrow \\
k \downarrow\end{array}$ & 3 & 4 & 5 & 6 \\
\hline 1 & 0.9111 & 0.8706 & 0.8580 & 0.8457 \\
2 & 0.9111 & 0.8598 & 0.8286 & 0.8234 \\
3 & -- & 0.8706 & 0.8348 & 0.8172 \\
4 & -- & -- & 0.8587 & 0.8372 \\
5 & -- & -- & -- & 0.8477 \\
\hline
\end{tabular}

Table 16: $C C E_{I A C_{\infty}}^{B}(k, m)$

\begin{tabular}{|c|cccc|}
\hline $\begin{array}{c}m \rightarrow \\
k \downarrow\end{array}$ & 3 & 4 & 5 & 6 \\
\hline 1 & 0.8815 & 0.7426 & 0.6143 & 0.5207 \\
2 & 0.8815 & 0.7468 & 0.6210 & 0.5386 \\
3 & -- & 0.7426 & 0.6337 & 0.5715 \\
4 & -- & -- & 0.6140 & 0.5330 \\
5 & -- & -- & -- & 0.5183 \\
\hline
\end{tabular}

\subsection{The selection of the Condorcet winner when one exists}

It is interesting to investigate the selection of the Condorcet winner when it exists as a member of the selected committee. For this issue, our results are displayed in Tables (17) to (21) where $C W_{I A C \infty}^{k-P}(k, m), C W_{I A C \infty}^{k-N P}(k, m)$, $C W_{I A C \infty}^{k-B}(k, m), C W_{I A C \infty}^{B}(k, m)$ and $C W_{I A C \infty}^{C C R}(k, m)$ denote the limiting probability under IAC as $n \rightarrow \infty$ that the $k$-Plurality rule, the $k$-Negative Plurality rule, the $k$-Borda rule, the Bloc rule, and the Chamberlin-Courant rule will respectively select the Condorcet winner when it exists for a given pair $(k, m)$. We draw some interesting observations from these tables. First, for each of the five multi-winner voting rules and a fixed number $m$ of competing candidates, the probability of selecting the Condorcet winner when it exists always 
increases as the target size $k$ of the committee increases from 1 to $m-1$. This result is expected because as the subset of candidates that has to be elected becomes larger the chance of the rule picking the Condorcet winner in this subset also increases. Second, it is worth mentioning that the $k$-Borda rule selects the Condorcet winner with certainty when $k=m-1$. The reason is that the Condorcet winner is never bottom ranked in the Borda ranking (see e.g., Fishburn and Gehrlein, 1976, Saari, 2000) and therefore the Condorcet winner is always selected by the $k$-Borda rule among the candidates ranked in the $m-1$ first positions. Third, for a given size $k$, the probability of every multi-winner rule selecting the Condorcet winner tends to decrease as the number $m$ of competing candidates increases. Finally, our results show that the use of the $k$-Borda rule significantly increases the probability of selecting the Condorcet winner when it exists, whereas the $k$-Plurality rule is the worst scenario according to this criteria. In other words, the replacement of the $k$ Borda rule with any of the other considered multi-winner rules significantly increases the risk of not electing the Condorcet winner as a member of the selected committee.

Table 17: $C_{I_{\text {IAC }}^{\infty}}^{C C R}(k, m)$
\begin{tabular}{|c|cccc|}
\hline$m \rightarrow$ & 3 & 4 & 5 & 6 \\
$k \downarrow$ & & & & \\
\hline 1 & 0.9111 & 0.8706 & 0.8580 & 0.8457 \\
2 & 0.9685 & 0.9324 & 0.9012 & 0.8803 \\
3 & -- & 0.9762 & 0.9457 & 0.9175 \\
4 & -- & -- & 0.9808 & 0.9556 \\
5 & -- & -- & -- & 0.9839 \\
\hline
\end{tabular}

Table 18: $C W_{I A C_{\infty}}^{k-B}(k, m)$

\begin{tabular}{|c|cccc|}
\hline $\begin{array}{c}m \rightarrow \\
k \downarrow\end{array}$ & 3 & 4 & 5 & 6 \\
\hline 1 & 0.9111 & 0.8706 & 0.8580 & 0.8457 \\
2 & 1 & 0.9962 & 0.9910 & 0.9875 \\
3 & -- & 1 & 0.9998 & 0.9994 \\
4 & -- & -- & 1 & 0.9999 \\
5 & -- & -- & -- & 1 \\
\hline
\end{tabular}

Table 19: $C W_{I A C_{\infty}}^{k-P}(k, m)$

Table 20: $C W_{I A C_{\infty}}^{B}(k, m)$

\begin{tabular}{|c|cccc|}
\hline $\begin{array}{c}m \rightarrow \\
k \downarrow\end{array}$ & 3 & 4 & 5 & 6 \\
\hline 1 & 0.8815 & 0.7426 & 0.6143 & 0.5207 \\
2 & 0.9685 & 0.9117 & 0.8334 & 0.7510 \\
3 & -- & 0.9762 & 0.9335 & 0.8763 \\
4 & -- & -- & 0.9808 & 0.9459 \\
5 & -- & -- & -- & 0.9839 \\
\hline
\end{tabular}

\begin{tabular}{|c|cccc|}
\hline $\begin{array}{c}m \rightarrow \\
k \downarrow\end{array}$ & 3 & 4 & 5 & 6 \\
\hline 1 & 0.8815 & 0.7426 & 0.6143 & 0.5207 \\
2 & 0.9704 & 0.9551 & 0.9199 & 0.8728 \\
3 & -- & 0.9773 & 0.9762 & 0.9709 \\
4 & -- & -- & 0.9818 & 0.9856 \\
5 & -- & -- & -- & 0.9847 \\
\hline
\end{tabular}




Table 21: $C W_{I A C_{\infty}}^{k-N P}(k, m)$
\begin{tabular}{|c|cccc|}
\hline$m \rightarrow$ & 3 & 4 & 5 & 6 \\
$k \downarrow$ & & & & \\
\hline 1 & 0.6296 & 0.5516 & 0.5104 & 0.4696 \\
2 & 0.9704 & 0.8450 & 0.7764 & 0.7179 \\
3 & -- & 0.9773 & 0.9175 & 0.8625 \\
4 & -- & -- & 0.9818 & 0.9427 \\
5 & -- & -- & -- & 0.9847 \\
\hline
\end{tabular}

3.4 The selection of the Condorcet loser when one exists

Note that, in single-winner elections, when the Condorcet loser is selected, this is referred to be an occurrence of the strong Borda paradox (see e.g., Diss and Doghmi, 2016, Diss and Gehrlein, 2012, Gehrlein and Lepelley, 2010, Kamwa and Valognes, 2017). It is well known that the Condorcet loser is never top-ranked in the Borda ranking (see e.g., Fishburn and Gehrlein, 1976, Saari, 2000). It is also well established that this outcome can occur with the Plurality rule and many other voting rules (see e.g., Felsenthal and Maoz, 1988, Gehrlein and Fishburn, 1978b, Gehrlein and Lepelley, 1998, Lepelley, 1993, Moyouwou and Tchantcho, 2017, Plassmann and Tideman, 2014). These studies have been conducted using either computer simulations or analytical computations under many assumptions regarding voters' preferences.

Our attention is drawn specifically in this section to evaluating the probability of selecting the Condorcet loser when it exists among the set of winners when a group of voters takes on the task of selecting a given number of candidates from a set of available candidates. Our results are displayed in Tables (22) to (26) where $C L_{I A C \infty}^{k-P}(k, m), C L_{I A C \infty}^{k-N P}(k, m), C L_{I A C \infty}^{k-B}(k, m), C L_{I A C \infty}^{B}(k, m)$ and $C L_{I A C \infty}^{C C R}(k, m)$ denote the limiting probability under IAC as $n \rightarrow \infty$ that the $k$-Plurality rule, the $k$-Negative Plurality rule, the $k$-Borda rule, the Bloc rule, and the Chamberlin-Courant rule will respectively select the Condorcet loser when it exists for a given pair $(k, m)$. Some observations can be drawn from these tables. First, for a fixed number of competing candidates $m$, the probability of selecting the Condorcet loser tends to decrease as the size $k$ of the committee increases. Second, for a fixed $k$, the probability of selecting the Condorcet loser tends to increase as the number of competing candidates $m$ increases. Finally, we can point out that for every pair $(k, m)$, the selection of the Condorcet loser under the $k$-Borda rule is relatively much less likely to be detected compared to the other rules. Overall, this probability only exceeds $15 \%$ for the pair $(k=5, m=6)$ under the $k$-Borda rule whereas it reaches $50 \%$ under the other rules. Finally, it is concluded that the use of 
rules like $k$-Plurality will clearly tend to maximize the likelihood of selecting the Condorcet loser among the set of winning candidates. Notice that in single-winner elections it has been established that the probabilities of selecting the Condorcet loser are maximized for voting rules like the Plurality rule and the Negative Plurality rule. The $k$-Plurality rule seems to be a bad choice not only in the single-winner case but also in the multi-winner scenario.

Table 22: $C_{I A C_{\infty}}^{C C R}(k, m)$
\begin{tabular}{|c|cccc|}
\hline$m \rightarrow$ & 3 & 4 & 5 & 6 \\
$k \downarrow$ & & & & \\
\hline 1 & 0 & 0 & 0 & 0 \\
2 & 0.3704 & 0.1307 & 0.0416 & 0.0138 \\
3 & -- & 0.4484 & 0.2006 & 0.0946 \\
4 & -- & -- & 0.4935 & 0.2586 \\
5 & -- & -- & -- & 0.5260 \\
\hline
\end{tabular}

Table 23: $C L_{I A C_{\infty}}^{k-B}(k, m)$
\begin{tabular}{|c|cccc|}
\hline$m \rightarrow$ & 3 & 4 & 5 & 6 \\
$k \downarrow$ & & & & \\
\hline 1 & 0 & 0 & 0 & 0 \\
2 & 0.0889 & 0.0038 & 0.0001 & 0.0000 \\
3 & -- & 0.1294 & 0.0087 & 0.0003 \\
4 & -- & -- & 0.1462 & 0.0125 \\
5 & -- & -- & -- & 0.1528 \\
\hline
\end{tabular}

Table 24: $C L_{I A C_{\infty}}^{k-P}(k, m)$

\begin{tabular}{|c|cccc|}
\hline $\begin{array}{c}m \rightarrow \\
k \downarrow\end{array}$ & 3 & 4 & 5 & 6 \\
\hline 1 & 0.0296 & 0.0227 & 0.0183 & 0.0147 \\
2 & 0.3704 & 0.1549 & 0.0824 & 0.0565 \\
3 & -- & 0.4484 & 0.2249 & 0.1379 \\
4 & -- & -- & 0.4935 & 0.2799 \\
5 & -- & -- & -- & 0.5260 \\
\hline
\end{tabular}

Table 25: $C L_{I A C_{\infty}}^{B}(k, m)$

\begin{tabular}{|c|cccc|}
\hline $\begin{array}{c}m \rightarrow \\
k \downarrow\end{array}$ & 3 & 4 & 5 & 6 \\
\hline 1 & 0.0296 & 0.0227 & 0.0183 & 0.0147 \\
2 & 0.1185 & 0.0449 & 0.0229 & 0.0145 \\
3 & -- & 0.2574 & 0.0808 & 0.0254 \\
4 & -- & -- & 0.3843 & 0.1279 \\
5 & -- & -- & -- & 0.4798 \\
\hline
\end{tabular}

Table 26: $C L_{I A C_{\infty}}^{k-N P}(k, m)$

\begin{tabular}{|c|cccc|}
\hline $\begin{array}{c}m \rightarrow \\
k \downarrow\end{array}$ & 3 & 4 & 5 & 6 \\
\hline 1 & 0.0315 & 0.0238 & 0.0199 & 0.0159 \\
2 & 0.1185 & 0.0883 & 0.0675 & 0.0529 \\
3 & -- & 0.2574 & 0.1670 & 0.1231 \\
4 & -- & -- & 0.3843 & 0.2491 \\
5 & -- & -- & -- & 0.4798 \\
\hline
\end{tabular}

\section{Concluding remarks}

The aim of this paper was to determine how often the outcome of various multi-winner voting rules might coincide for the selection of a same unique committee of a given size. We focused on five well-studied rules: 
the Chamberlin-Courant rule, the $k$-Plurality rule, the $k$-Borda rule, the $k$ Negative Plurality rule and the Bloc rule. We focused on voting situations with $m=3,4,5,6$ candidates and committees of size $1<k<m-1$. Some results are very clear from the calculated values of our probabilities. Over the range of scenarios that have been considered in this paper, we found that in most of the cases one should expect to observe an agreement between any pair of the considered multi-winner rules, with greater probability in the asymptotic case as the number of voters tends to infinity. We also drew an interesting comparison between every multi-winner rule on the basis of their propensity to follow certain principles based on the Condorcet idea. One of the possibilities was, for instance, to examine the election of the Condorcet committee $a$ la Gehrlein when it exists which guarantees a committee with undominated members. The selection of the Condorcet winner/loser, when it exists, among the members of the elected committee, was also examined in this paper. We have been able to show that in general the $k$-Borda rule performs better than the four other multi-winner voting rules, whereas the $k$-Plurality rule and the $k$-Negative Plurality rule seem to be the worst scenarios.

Since we have studied the particular case of scoring rules, the extension of these results to multistage elimination scoring rules or other variants of Chamberlin-Courant's rule remains open. More particularly, a similar analysis can be achieved with the well-known Single Transferable Vote (STV). The most studied variant of STV in the multi-winner setting works as follows: Each voter has a single vote that is initially allocated to her most-preferred candidate. In each round, votes are aggregated and if there is a candidate, say $a$, whose Plurality score is greater than the well-known Droop quota $q$ which is fixed before the election, the following procedure is used: First, candidate $a$ is selected in the winning committee; second, the $q$ votes where $a$ is ranked first are deleted; and finally candidate $a$ is removed from all the remaining votes. If each candidate's Plurality score is less than $q$, the candidate with the lowest Plurality score is eliminated from all votes. This procedure is repeated until the election of the target committee. It would be very interesting to study the probability of the agreement between this rule and the five multiwinner voting rules that we have considered in this paper, but discussion of this rule and other multistage elimination scoring rules is beyond the scope of this paper. Notice, finally, that results for more than six candidates would have allowed us to draw more accurate conclusions. However, obtaining such results runs up against the limits of probability calculations under the IAC assumption, even with computer simulations. 


\section{Appendix}

Recall that the Convex code (MAPLE) has been used to obtain the results for 3 and 4 candidates, except for the probabilities displayed in Table 11 for which we used Normaliz. All our results for 5 and 6 candidates have been obtained using computer simulations (MATLAB). We provide the three codes in the following sections. In order to illustrate our calculations, some useful comments are given in the codes. For further information, the reader is referred to the original papers listed in Section 2.3.

\subsection{Use of Convex}

To illustrate the use of Convex, suppose that we want to calculate the probability of the agreement between the $k$-Plurality rule and the $k$-Negative Plurality rule for the pair $(k=3, m=4)$.

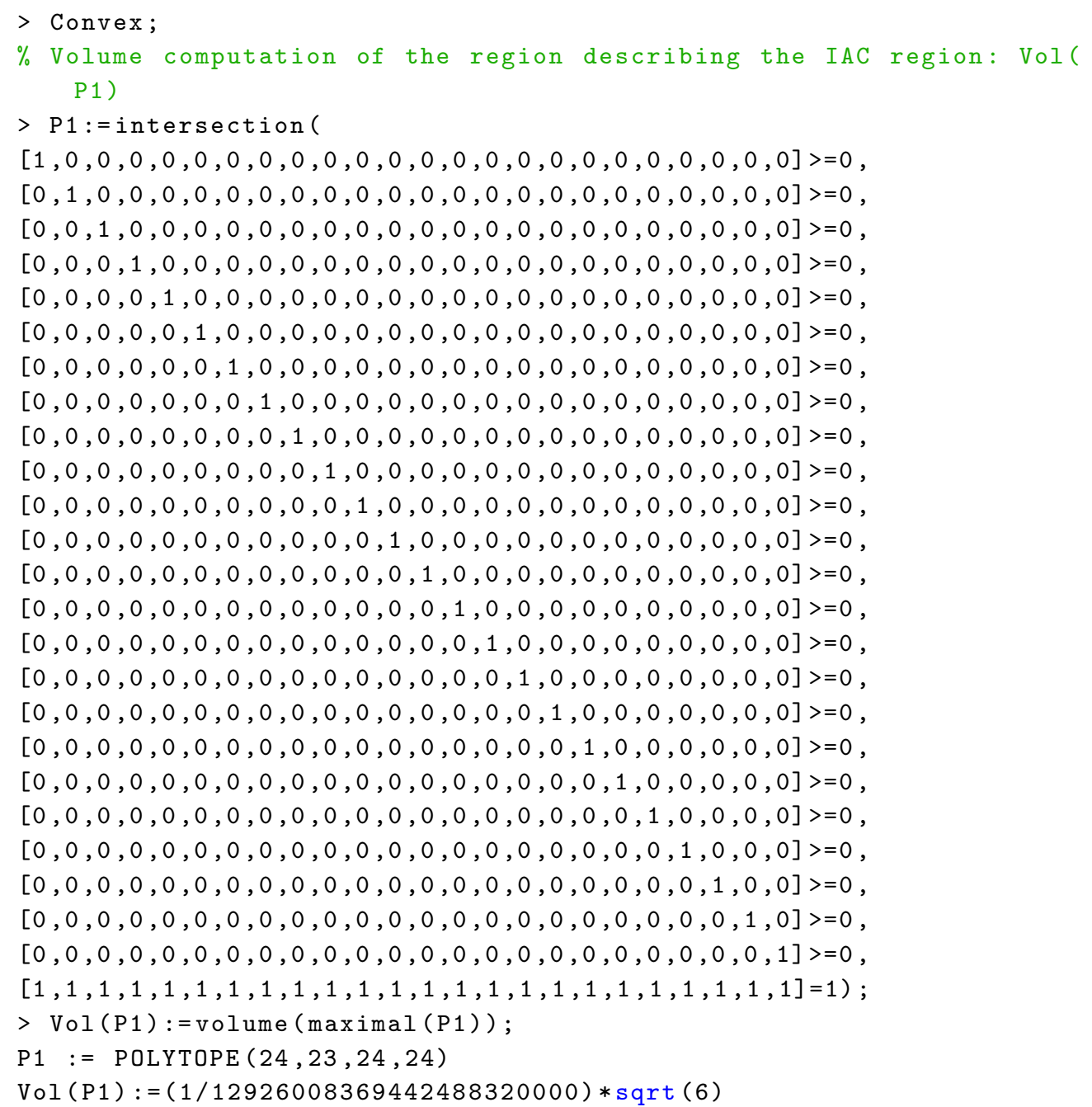




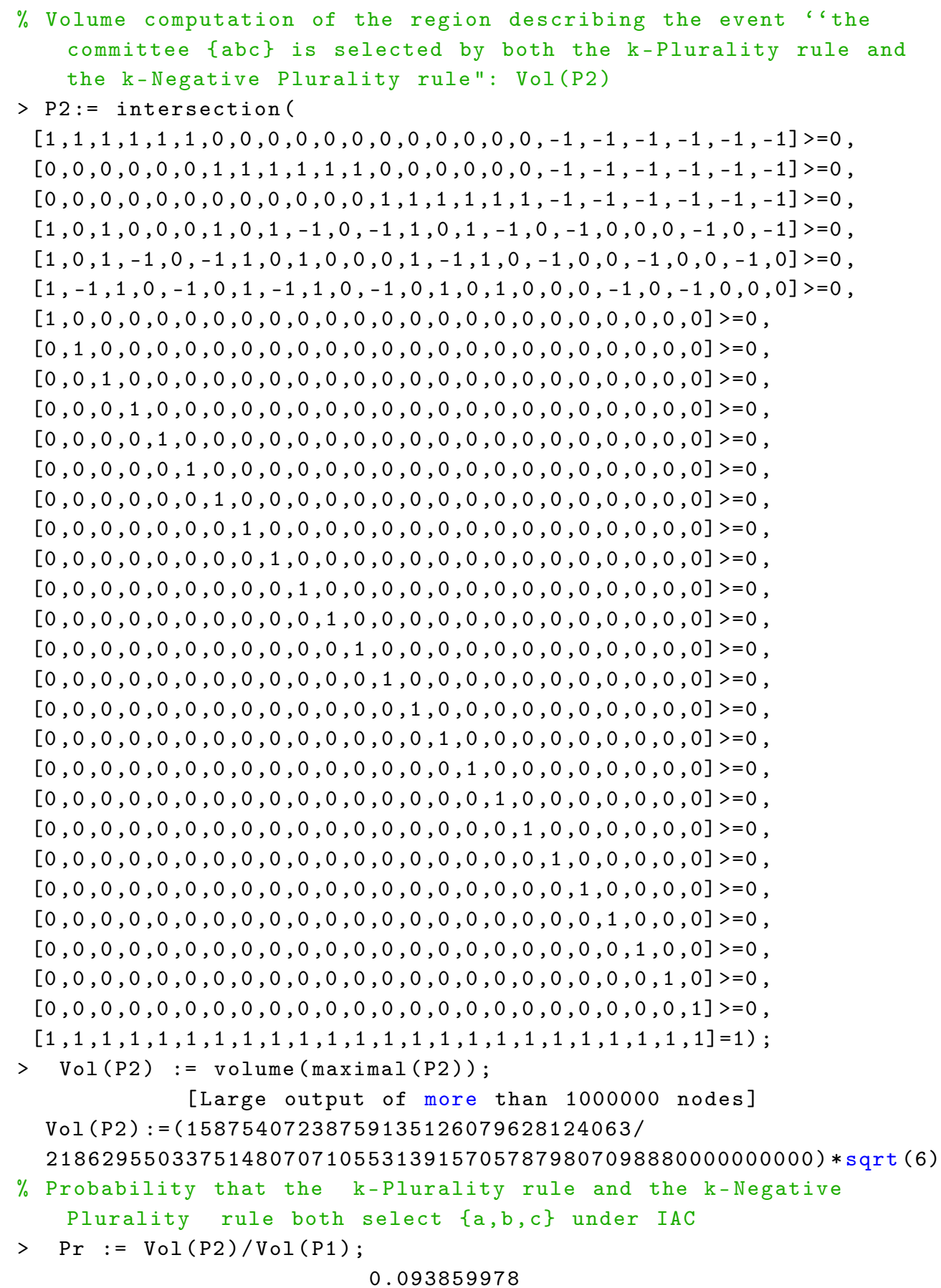

Since there are 4 possible committees of size 3 , the probability that the $k$ Plurality rule and the $k$-Negative Plurality rule agree for the pair $(k=3, m=$ 4 ) is simply: $4 \times 0.093859978=0.3754399101$. 


\subsection{Use of Normaliz}

To illustrate the use of Normaliz, suppose that we want to find the probability of the agreement between all the considered multi-winner rules for the pair $(k=1, m=4)$. It is useful here to recall that for $k=1$, the Bloc rule and the $k$-Borda rule are respectively equivalent to the $k$-Plurality rule and the Chamberlin-Courant rule. Thus, the conditions under which a certain candidate, say $a$, is the winner under all the considered multi-winner rules are reduced to 9 linear inequalities (instead of 15) described in the Normaliz code below. Note that in Normaliz, the whole region of IAC is described by a lattice normalized volume in which the unit simplex has volume 1.

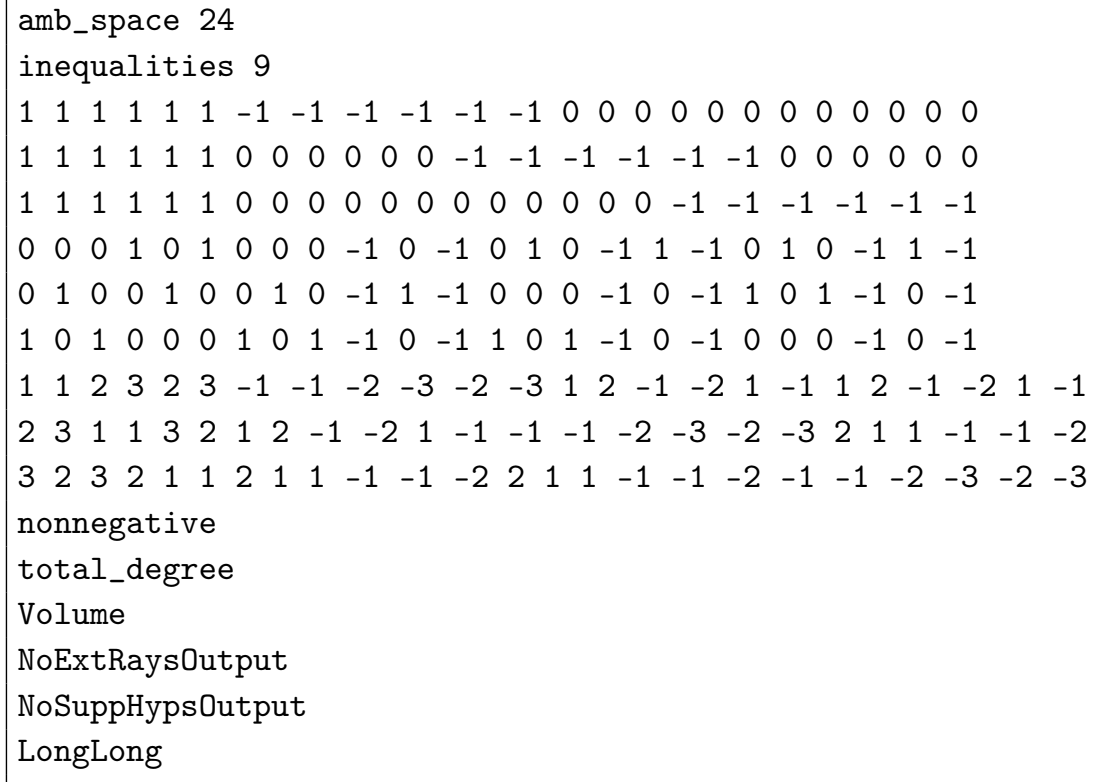

From the output file we obtain the quantity:

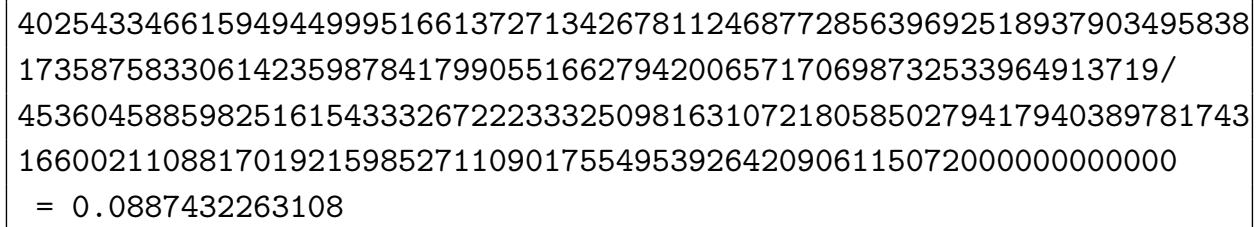

Since there are 4 candidates, the probability that all the considered multiwinner rules agree for the pair $(k=1, m=4)$ is: $4 \times 0.0887432263108=$ 0.354972905 . 
5.3 Use of computer simulations: MATLAB code

To illustrate the use of computer simulations, suppose that we want to estimate the probability of the agreement between the $k$-Plurality rule and the $k$-Borda rule for the pair $(k=1, m=5)$.

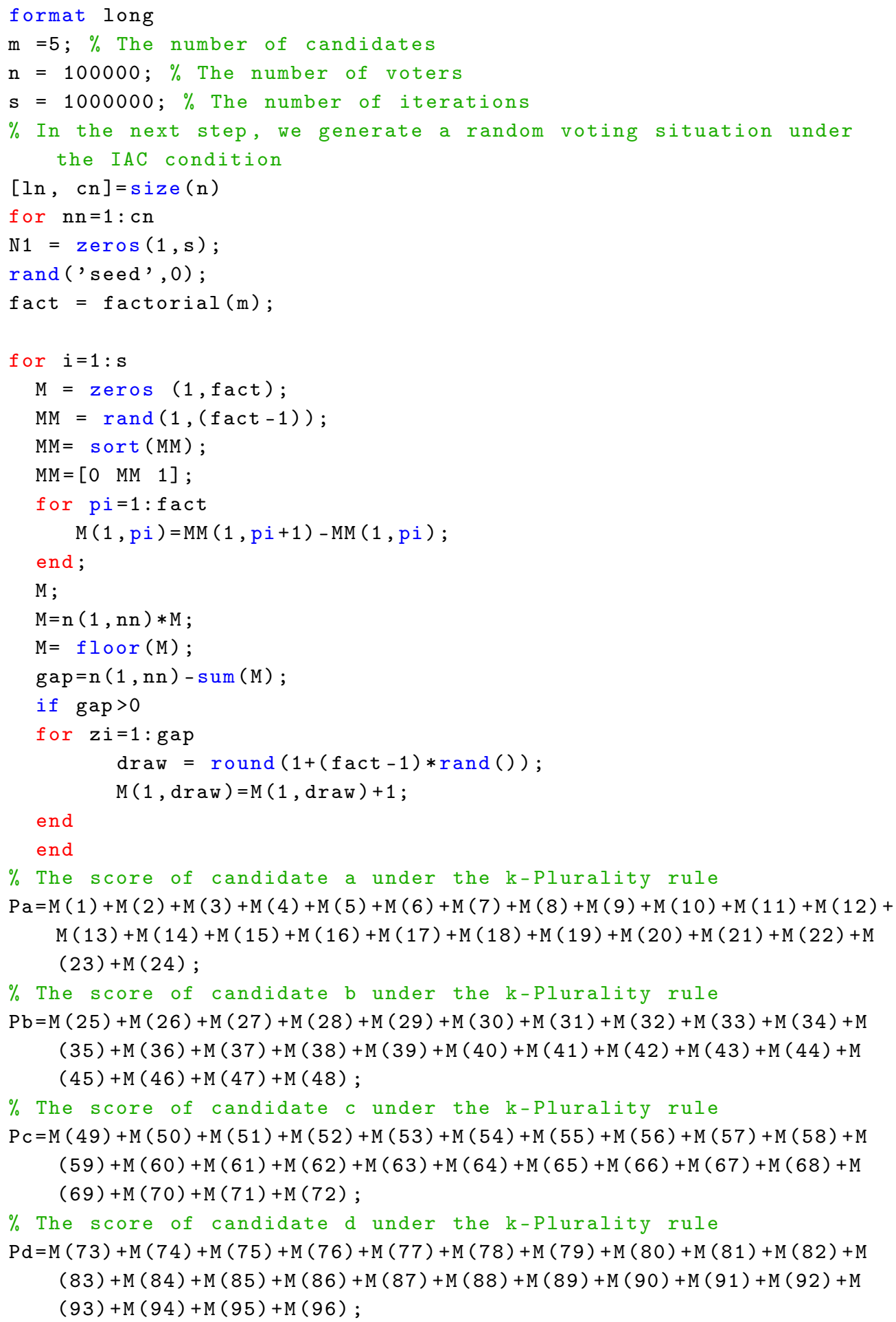


$\%$ The score of candidate e under the k-Plurality rule

$P e=M(97)+M(98)+M(99)+M(100)+M(101)+M(102)+M(103)+M(104)+M(105)+M$ $(106)+M(107)+M(108)+M(109)+M(110)+M(111)+M(112)+M(113)+M(114)+$ $M(115)+M(116)+M(117)+M(118)+M(119)+M(120) ;$

$\%$ The score of candidate a under the $\mathrm{k}$-Borda rule

$\mathrm{Ba}=4 * \mathrm{M}(1)+4 * \mathrm{M}(2)+4 * \mathrm{M}(3)+4 * \mathrm{M}(4)+4 * \mathrm{M}(5)+4 * \mathrm{M}(6)+4 * \mathrm{M}(7)+4 * \mathrm{M}(8)+4 * \mathrm{M}(9)$ $+4 * \mathrm{M}(10)+4 * \mathrm{M}(11)+4 * \mathrm{M}(12)+4 * \mathrm{M}(13)+4 * \mathrm{M}(14)+4 * \mathrm{M}(15)+4 * \mathrm{M}(16)+4 * \mathrm{M}$ $(17)+4 * M(18)+4 * M(19)+4 * M(20)+4 * M(21)+4 * M(22)+4 * M(23)+4 * M(24)$ $+3 * M(25)+3 * M(26)+3 * M(27)+3 * M(28)+3 * M(29)+3 * M(30)+2 * M(31)+2 * M$ $(32)+M(33)+M(35)+2 * M(37)+2 * M(38)+M(39)+M(41)+2 * M(43)+2 * M(44)+M$ $(45)+M(47)+3 * M(49)+3 * M(50)+3 * M(51)+3 * M(52)+3 * M(53)+3 * M(54)+2 * M$ $(55)+2 * M(56)+M(57)+M(59)+2 * M(61)+2 * M(62)+M(63)+M(65)+2 * M(67)$ $+2 * M(68)+M(69)+M(71)+3 * M(73)+3 * M(74)+3 * M(75)+3 * M(76)+3 * M(77)$ $+3 * M(78)+2 * M(79)+2 * M(80)+M(81)+M(83)+2 * M(85)+2 * M(86)+M(87)+M$ $(89)+2 * M(91)+2 * M(92)+M(93)+M(95)+3 * M(97)+3 * M(98)+3 * M(99)+3 * M$ $(100)+3 * M(101)+3 * M(102)+2 * M(103)+2 * M(104)+M(105)+M(107)+2 * M$ $(109)+2 * M(110)+M(111)+M(113)+2 * M(115)+2 * M(116)+M(117)+M(119)$;

$\%$ The score of candidate $b$ under the $\mathrm{k}-\mathrm{B}$ orda rule

$\mathrm{Bb}=3 * \mathrm{M}(1)+3 * \mathrm{M}(2)+3 * \mathrm{M}(3)+3 * \mathrm{M}(4)+3 * \mathrm{M}(5)+3 * \mathrm{M}(6)+2 * \mathrm{M}(7)+2 * \mathrm{M}(8)+\mathrm{M}(9)+\mathrm{M}$ $(11)+2 * M(13)+2 * M(14)+M(15)+M(17)+2 * M(19)+2 * M(20)+M(21)+M(23)$ $+4 * \mathrm{M}(25)+4 * \mathrm{M}(26)+4 * \mathrm{M}(27)+4 * \mathrm{M}(28)+4 * \mathrm{M}(29)+4 * \mathrm{M}(30)+4 * \mathrm{M}(31)+4 * \mathrm{M}$ $(32)+4 * M(33)+4 * M(34)+4 * M(35)+4 * M(36)+4 * M(37)+4 * M(38)+4 * M(39)$ $+4 * M(40)+4 * M(41)+4 * M(42)+4 * M(43)+4 * M(44)+4 * M(45)+4 * M(46)+4 * M$ $(47)+4 * M(48)+2 * M(49)+2 * M(50)+M(51)+M(53)+3 * M(55)+3 * M(56)+3 * M$ $(57)+3 * M(58)+3 * M(59)+3 * M(60)+M(61)+2 * M(63)+2 * M(64)+M(66)+M(67)$ $+2 * M(69)+2 * M(70)+M(72)+2 * M(73)+2 * M(74)+M(75)+M(77)+3 * M(79)+3 * M$ $(80)+3 * M(81)+3 * M(82)+3 * M(83)+3 * M(84)+M(85)+2 * M(87)+2 * M(88)+M$ $(90)+M(91)+2 * M(93)+2 * M(94)+M(96)+2 * M(97)+2 * M(98)+M(99)+M(101)$ $+3 * M(103)+3 * M(104)+3 * M(105)+3 * M(106)+3 * M(107)+3 * M(108)+M(109)$ $+2 * M(111)+2 * M(112)+M(114)+M(115)+2 * M(117)+2 * M(118)+M(120) ;$

$\%$ The score of candidate $c$ under the $\mathrm{k}-\mathrm{B}$ orda rule

$\mathrm{BC}=2 * \mathrm{M}(1)+2 * \mathrm{M}(2)+\mathrm{M}(3)+\mathrm{M}(5)+3 * \mathrm{M}(7)+3 * \mathrm{M}(8)+3 * \mathrm{M}(9)+3 * \mathrm{M}(10)+3 * \mathrm{M}(11)+3 *$ $M(12)+M(13)+2 * M(15)+2 * M(16)+M(18)+M(19)+2 * M(21)+2 * M(22)+M(24)$ $+2 * M(25)+2 * M(26)+M(27)+M(29)+3 * M(31)+3 * M(32)+3 * M(33)+3 * M(34)$ $+3 * M(35)+3 * M(36)+M(37)+2 * M(39)+2 * M(40)+M(42)+M(43)+2 * M(45)+2 * M$ $(46)+M(48)+4 * M(49)+4 * M(50)+4 * M(51)+4 * M(52)+4 * M(53)+4 * M(54)+4 * M$ $(55)+4 * M(56)+4 * M(57)+4 * M(58)+4 * M(59)+4 * M(60)+4 * M(61)+4 * M(62)$ $+4 * M(63)+4 * M(64)+4 * M(65)+4 * M(66)+4 * M(67)+4 * M(68)+4 * M(69)+4 * M$ $(70)+4 * M(71)+4 * M(72)+M(73)+2 * M(75)+2 * M(76)+M(78)+M(79)+2 * M(81)$ $+2 * M(82)+M(84)+3 * M(85)+3 * M(86)+3 * M(87)+3 * M(88)+3 * M(89)+3 * M(90)$ $+M(92)+M(94)+2 * M(95)+2 * M(96)+M(97)+2 * M(99)+2 * M(100)+M(102)+M$ $(103)+2 * M(105)+2 * M(106)+M(108)+3 * M(109)+3 * M(110)+3 * M(111)+3 * M$ $(112)+3 * M(113)+3 * M(114)+M(116)+M(118)+2 * M(119)+2 * M(120)$;

$\%$ The score of candidate d under the $\mathrm{k}-\mathrm{Borda}$ rule

$B d=M(1)+2 * M(3)+2 * M(4)+M(6)+M(7)+2 * M(9)+2 * M(10)+M(12)+3 * M(13)+3 * M$ $(14)+3 * M(15)+3 * M(16)+3 * M(17)+3 * M(18)+M(20)+M(22)+2 * M(23)+2 * M$ $(24)+M(25)+2 * M(27)+2 * M(28)+M(30)+M(31)+2 * M(33)+2 * M(34)+M(36)$ $+3 * M(37)+3 * M(38)+3 * M(39)+3 * M(40)+3 * M(41)+3 * M(42)+M(44)+M(46)$ $+2 * M(47)+2 * M(48)+M(49)+2 * M(51)+2 * M(52)+M(54)+M(55)+2 * M(57)+2 * M$ $(58)+M(60)+3 * M(61)+3 * M(62)+3 * M(63)+3 * M(64)+3 * M(65)+3 * M(66)+M$ $(68)+M(70)+2 * M(71)+2 * M(72)+4 * M(73)+4 * M(74)+4 * M(75)+4 * M(76)+4 * M$ $(77)+4 * M(78)+4 * M(79)+4 * M(80)+4 * M(81)+4 * M(82)+4 * M(83)+4 * M(84)$ $+4 * M(85)+4 * M(86)+4 * M(87)+4 * M(88)+4 * M(89)+4 * M(90)+4 * M(91)+4 * M$ 


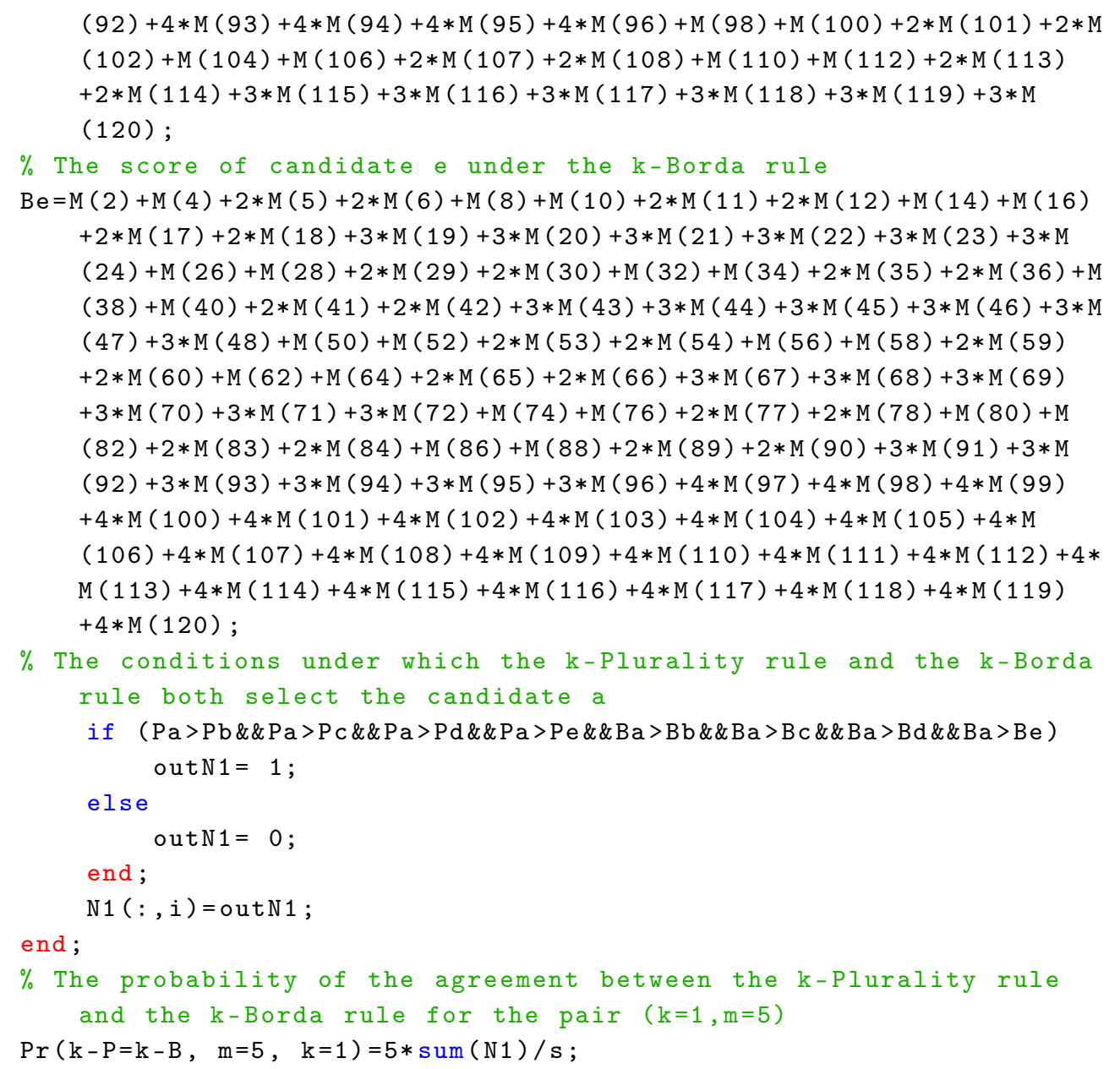

\section{References}

Aziz, H., Brill, M., Conitzer, V., Elkind, E., Freeman, R., and Walsh, T. (2017) Justified representation in approval-based committee voting. Social Choice and Welfare 48(2): 461-485.

Aziz, H., Gaspers, S., Mattei, N., Narodytska, N., and Walsh, T. (2013) Ties matter: Complexity of manipulation when tie-breaking with a random vote. In Proc. of 27th AAAI Conference, pages 74-80. AAAI Press.

Barberà, S., and Coelho, D. (2008) How to choose a non-controversial list with $k$ names. Social Choice and Welfare 31: 79-96.

Betzler, N., Slinko, A., and Uhlmann, J. (2013) On the computation of fully proportional representation. Journal of Artificial Intelligence Research 47: 475-519.

Bock, H., Dayb, W., and McMorris, F. (1998) Consensus rules for committee elections. Mathematical Social Sciences 35(3): 219-232. 
Brams, S.J. (2008) Mathematics and Democracy: Designing Better Voting and Fair-Division Procedures. Princeton University Press.

Brams, S.J. and Brill, M. (2018) The excess method: A multiwinner approval voting procedure to allocate wasted votes. New York University.

Brams, S.J., Kilgour, D.M., and Potthoff, R.F. (2019) Multiwinner approval voting: an apportionment approach. Public Choice 178(1-2): 67-93.

Brams, S.J., Kilgour, D.M., and Sanver, M.R. (2005) A minimax procedure for electing committees. Public Choice 132(34): 401-420.

Brams, S.J., Kilgour, D.M., and Sanver, M.R. (2006) How to elect a representative committee using approval balloting. In: Simeone B., Pukelsheim F., editors. Mathematics and Democracy. Springer; Berlin. pp. 83-95. (Studies in Choice and Welfare).

Brill, M., Laslier, J.-F., and Skowron, P. (2018) Multiwinner approval rules as apportionment methods. Journal of Theoretical Politics 30(3): 358-382.

Bruns, W., Ichim, B., Römer, T., Sieg, R., \& Söger, C. (2017) Normaliz: Algorithms for rational cones and affine monoids. Available at http://normaliz.uos.de.

Bruns, W., Ichim, B., \& Söger, C. (2019) Computations of volumes and Ehrhart series in four candidates elections. Annals of Operations Research. Forthcoming.

Bubboloni, D., Diss, M., and Gori, M. (2018) Extensions of the Simpson voting rule to the committee selection setting. WP 1813, GATE Lyon Saint-Etienne.

Cervone, D., Gehrlein, W.V., and Zwicker, W. (2005) Which scoring rule maximizes Condorcet efficiency under IAC? Theory and Decision 58: 145-185.

Chamberlin, J.R., and Courant, P.N. (1983) Representative Deliberations and Representative Decisions: Proportional Representation and the Borda Rule. The American Political Science Review 77(3): 718-733.

Debord, B. (1993) Prudent $k$-choice functions: Properties and algorithms. Mathematical SocialSciences 26: 63-77.

Diss, M., and Doghmi, A. (2016) Multi-winner scoring election methods: Condorcet consistency and paradoxes. Public Choice 169: 97-116

Diss, M., and Gehrlein, W.V. (2015) The true impact of voting rule selection on Condorcet efficiency. Economics Bulletin 35: 2418-2426.

Diss, M., and Gehrlein, W.V. (2012) Borda's paradox with weighted scoring rules. Social Choice and Welfare 38: 121-136. 
Dodgson C.L (1884) The principles of parliamentary representation. Harrison and Sons Publisher, London.

Dodgson C.L (1876) A Method of Taking Votes on More than Two Issues. Clarendon Press, Oxford.

Droop H. (1881) On methods of electing representatives. Journal of the Statistical Society of London 44(2): 141-202.

Dummett, M. (1984) Voting Procedures. Oxford, Clarendon Press.

Elkind, E., Faliszewski P., Skowron, P., and Slinko, A. (2017) Properties of multiwinner voting rules. Social Choice and Welfare 48(3): 599-632.

Elkind, E., Lang, J., and Saffidine, A. (2011) Choosing collectively optimal sets of alternatives based on the Condorcet criterion. Proceedings IJCAI11, pp. 186-191.

Elkind, E., Lang, J., and Saffidine, A. (2015) Condorcet winning sets. Social Choice and Welfare 44(3): 493-517.

El Ouafdi, A., Lepelley, D., and Smaoui, H. (2018) Probabilities of electoral outcomes in four-candidate elections. Preprint.

Franz, M. (2016) Convex - a Maple package for convex geometry, version 1.2 available at http://www-home.math.uwo.ca/ mfranz/convex/.

Faliszewski, P., Skowron, P., Slinko, A., and Talmon, N. (2017) Multiwinner Rules on Paths From $k$-Borda to Chamberlin-Courant. Proceedings IJCAI17, pp. 192-198.

Faliszewski, P., Skowron, P., Slinko, A. and Talmon, N. (2016) Committee Scoring Rules: Axiomatic Classification and Hierarchy. Proceedings of the Twenty-Fifth International Joint Conference on Artificial Intelligence (IJCAI-16).

Faliszewski, P., Skowron, P., Slinko, A. and Talmon, N. (2018) Multiwinner analogues of the Plurality rule: Axiomatic and algorithmic perspectives. Social Choice and Welfare, 51(3): 513-550.

Felsenthal, D.S., and Maoz, Z. (1988) A Comparative Analysis of Sincere and Sophisticated Voting Under the Plurality and Approval Voting Procedures. Behavioral Science 33: 116-130.

Fishburn, P.C. (1981) An analysis of simple voting systems for electing committees. SIAM Journal on Applied Mathematics 41: 499-502.

Fishburn, P.C. (1977) Condorcet social choice functions. SIAM Journal of Applied Mathematics, 33: 469-489. 
Fishburn, P.C., and Gehrlein, W.V. (1976) Borda's Rule, Positional Voting, and Condorcet's Simple Majority Principle. Public Choice 28:, 79-88.

Gehrlein, W.V. (1985) The Condorcet criterion and committee selection. Mathematical Social Sciences 10: 199-209.

Gehrlein, W.V. (1999) On the Probability that all Weighted Scoring Rules Elect the Condorcet Winner. Quality and Quantity 33(1):77-84.

Gehrlein, W.V., and Fishburn, P.C. (1978a) Coincidence Probabilities for Simple Majority and Positional Voting Rules. Social Science Research 7: 272-283.

Gehrlein, W.V., and Fishburn, P.C. (1978b) Probabilities of elections outcomes for large electorates. Journal of Economic Theory 19: 38-49.

Gehrlein, W.V., and Fishburn, P.C. (1976) The probability of the paradox of voting: A computable solution. Journal of Economic Theory 13: 14-25.

Gehrlein, W.V., and Lepelley, D. (2017) Elections, Voting Rules and Paradoxical Outcomes. Springer-Verlag.

Gehrlein, W.V., and Lepelley, D. (2011) Voting paradoxes and group coherence. Springer, Berlin/Heidelberg.

Gehrlein, W.V., and Lepelley, D. (2010) On the probability of observing Borda's Paradox. Social Choice and Welfare 35: 1-23.

Gehrlein, W.V., and Lepelley, D. (1998) The Condorcet efficiency of approval voting and the probability of electing the Condorcet loser. Journal of Mathematical Economics 29: 271-283.

Gehrlein, W.V., Lepelley, D., and Moyouwou, I. (2015) Voters' preference diversity, concepts of agreement and Condorcet's paradox. Quality \& Quantity $49(6): 2345^{-2368 . ~}$

Guilbaud, G.T. (1952) Les théories de l'intérêt général et le problème logique de l'agrégation. Economie Appliquée 5: 501-584.

Kamwa, E. (2017) On stable rules for selecting committees. Journal of Mathematical Economics 70: 36-44.

Kamwa, E., and Merlin, V. (2014) Some remarks on the Chamberlin-Count rule. Mimeo, Université de Caen.

Kamwa, E., and Merlin, V. (2015) Scoring rules over subsets of alternatives: Consistency and paradoxes. Journal of Mathematical Economics 61: 130138.

Kamwa, E., and Merlin, V. (2018) Coincidence of Condorcet Committees. Social Choice and Welfare 50(1): 171-189. 
Kamwa, E., and Valognes, F. (2017) Scoring rules and preference restrictions: The Strong Borda Paradox revisited. Revue d'Economie Politique 127(3): 375-395.

Kaymak, B., and Sanver, M.R. (2003) Sets of alternatives as Condorcet winners. Social Choice and Welfare 20: 477-494.

Kilgour, M. (2018) Multi-winner voting. Estudios de Economia Applicada 36(1): 167-180.

Kilgour, M. (2010) Approval balloting for multi-winner elections. In: Laslier, J.-F., Sanver, M.R. (eds) Handbook on approval voting. Springer, Berlin, pp 105-124.

Kilgour, M., and Marshall, E. (2012) Approval balloting for fixed-size committees. In Felsenthal, Dan S., and Machover, M. editors, Electoral Systems: Paradoxes, Assumptions, and Procedures, 305-326. Springer Heidelberg.

Klamler, C. (2004) The Dodgson Ranking and Its Relation to Kemeny's Method and Slater's Rule. Social Choice and Welfare 23: 91-102.

Klamler, C. (2003) A Comparison of the Dodgson Method and the Copeland Rule. Economics Bulletin 4(8): 1-7.

Lepelley, D. (1993) On the probability of electing the Condorcet loser. Mathematical Social Sciences 25: 105-116.

$\mathrm{Lu}, \mathrm{T}$., and Boutilier, C. (2011) Budgeted social choice: From consensus to personalized decision making. In Proceedings of IJCAI-11: 280-286.

Mathur, A. and Bhattacharyya, A. (2017) On the Gap between Outcomes of Voting Rules. In: Proc. of the 16th International Conference on Autonomous Agents and Multiagent Systems (AAMAS 2017), S. Das, E. Durfee, K. Larson, M. Winikoff (eds.).

Mattei, N., Narodytska, N., and Walsh T. (2014) How hard is it to control an election by breaking ties? In Proc. of 21st ECAI, pages 1067-1068.

Merlin, V., Tataru, M., and Valognes, F. (2000) On the probability that all decision rules select the same winner. Journal of Mathematical Economics 33: 183-207.

Moyouwou, I., and Tchantcho, H. (2017) A note on Approval Voting and electing the Condorcet loser. Mathematical Social Sciences 89: 70-82.

Obraztsova, S., Elkind, E., and Hazon, N. (2011) Ties matter: Complexity of voting manipulation revisited. In Proc. of 1oth AAMAS Conference, pages $71-78$. 
Nurmi, H. (1988) Discrepancies in the Outcomes Resulting From Different Voting Schemes. Theory and Decision 25: 193-208.

Nurmi, H. (1987) Comparing Voting Systems. Reidel.

Plassmann, F., and Tideman, N. (2014) How Frequently Do Different Voting Rules Encounter Voting Paradoxes in Three-candidate elections? Social Choice and Welfare 42: 31-75.

Potthoff, R.F., and Brams S.J. (1998) Proportional representation: Broadening the options. Journal of Theoritical Politics 10(2): 147-178.

Procaccia, A.D., Rosenschein, J.S., and Zohar, A. (2008) On the complexity of achieving proportional representation. Social Choice and Welfare 30: 353362 .

Ratliff, T.C. (2006) Selecting committees. Public Choice 126: 343-355.

Ratliff, T.C. (2003) Some startling inconsistencies when electing committees. Social Choice and Welfare 21: 433-454.

Ratliff, T.C. (2002) A Comparison of Dodgson's Method and the Borda Count. Economic Theory, 20: 357-372.

Ratliff, T.C. (2001) A Comparison of Dodgson's Method and Kemeny's Rule. Social Choice and Welfare 18: 79-90.

Regenwetter, M. and Grofman, B. (1998) Approval voting, Borda winners and Condorcet winners: evidence from seven elections. Management Science 44: 520-533.

Saari, D.G. (2000) Mathematical structure of voting paradoxes I. Pairwise votes. Economic Theory 15: 1-53.

Skowron, P., Faliszewski, P., and Slinko, A. (2016) Axiomatic Characterization of Committee Scoring Rules. Preprint arXiv: 1604.01529

Skowron, P., Faliszewski, P., and Slinko, A. (2013) Achieving fully proportional representation: Approximability results. Preprint arXiv: 1312.4026.

Skowron, P., Yu, L., Faliszewski, P., and Elkind, E. (2015) The complexity of fully proportional representation for single-crossing electorates. Theoretical Computer Science 569: 43-57.

Sterne, S. (1871) On representative government and personal representation. Philadelphia: J.B. Lippincott.

Young, H.P (1974) An axiomatization of the Borda rule. Journal of Economic Theory 9: 43-52. 